\title{
Sketch of Solutions
}

\subsection{Basic Elements}

\subsubsection{Fundamental Terms}

1. (a) We have

$$
V_{0}=\frac{\mathrm{E}\left[{\widetilde{F C F_{1}}}_{1}\right]}{1+\kappa_{0,1}}+\frac{F C F_{2}}{\left(1+r_{f}\right)^{2}}=\frac{100}{1.10}+\frac{100}{1.05^{2}} \approx 181.61 .
$$

The resulting cost of capital is

$$
k_{0}=\frac{\mathrm{E}\left[\widetilde{F C F}_{1}+V_{1}\right]}{V_{0}}-1 \approx \frac{100+\frac{100}{1.05}}{181.61} \approx 7.5028 \% .
$$

(b) We have

$$
V_{0}=\frac{\mathrm{E}\left[\widetilde{F C F}_{1}\right]}{1+k_{0}}+\frac{F C F_{2}}{\left(1+k_{0}\right)\left(1+r_{f}\right)}=\frac{100}{1.10}+\frac{100}{1.10 \cdot 1.05} \approx 177.49
$$

The discount rate is then

$$
\kappa_{0,1}=\frac{\mathrm{E}\left[{\widetilde{F C F_{1}}}_{1}\right]}{V_{0}-\frac{F C F_{2}}{\left(1+r_{f}\right)^{2}}}-1 \approx \frac{100}{177.49-\frac{100}{1.05^{2}}} \approx 15.2257 \% .
$$

2. Assume we have

$$
\left(1+\kappa_{t, t+1}\right)\left(1+\kappa_{t+1, t+2}\right)<\left(1+\kappa_{t, t+2}\right)^{2} .
$$


Then the investor should do the following. At time $t$ she invests $\frac{1}{\left(1+\kappa_{t, t+2}\right)^{2}}$ in the firm to get one dollar of cash flow at time $t+2$.

The amount needed is borrowed in the market: At time $t+1$ (tomorrow) she borrows $\frac{1}{1+\kappa_{t+1, t+2}}$ and has to pay back one dollar at time $t+2$. At time $t$ she again borrows $\frac{1}{\left(1+\kappa_{t, t+1}\right)\left(1+\kappa_{t+1, t+2}\right)}$ to pay back $\frac{1}{1+\kappa_{t+1, t+2}}$ at time $t+1$. Mind that both borrowing strategies do not require any net cash flows at time $t+1$ and $t+2$.

The first strategy requires an investment of $\frac{1}{\left(1+\kappa_{t, t+2}\right)^{2}}$ and the second one gives a payment of $\frac{1}{\left(1+\kappa_{t, t+1}\right)\left(1+\kappa_{t+1, t+2)}\right.}$ which is, by assumption, more. By investing again and again the investor will get infinitely rich.

If

$$
\left(1+\kappa_{t, t+1}\right)\left(1+\kappa_{t+1, t+2}\right)>\left(1+\kappa_{t, t+2}\right)^{2},
$$

a reverse strategy yields the arbitrage opportunity.

\subsubsection{Conditional Expectation}

1. This is straightforward, see Fig. 7.1.

2. The expectation is evaluated as

$$
\begin{aligned}
\mathrm{E}\left[\mathrm{E}_{Q}\left[\overline{F C F}_{2} \mid \mathcal{F}_{1}\right] \mid \mathcal{F}_{0}\right]= & \frac{1}{2}(0.1 \cdot 145.2+0.9 \cdot 121) \\
& +\frac{1}{2}(0.1 \cdot 121+0.9 \cdot 100.6)=113.03
\end{aligned}
$$

Fig. 7.1 Cash flows in future

193.6 periods

145.2

133.1

96.8

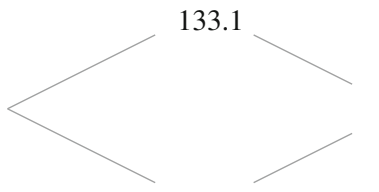

121

110.8

145.2

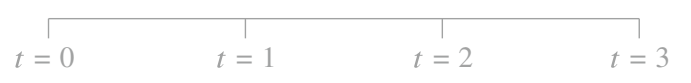


and

$$
\begin{aligned}
\mathrm{E}_{Q}\left[\mathrm{E}\left[{\widetilde{F C F_{2}}}_{2} \mid \mathcal{F}_{1}\right] \mid \mathcal{F}_{0}\right]=0.1\left(\frac{1}{2}\right. & \left.\cdot 145.2+\frac{1}{2} \cdot 121\right) \\
& +0.9\left(\frac{1}{2} \cdot 121+\frac{1}{2} \cdot 100.6\right)=113.03
\end{aligned}
$$

If we now change the order of expectation, we get

$$
\begin{aligned}
\mathrm{E}\left[\mathrm{E}_{Q}\left[\widetilde{F C F}_{2} \mid \mathcal{F}_{1}\right] \mid \mathcal{F}_{0}\right]= & \frac{1}{2}(0.1 \cdot 145.2+0.9 \cdot 120) \\
& +\frac{1}{2}(0.1 \cdot 122+0.9 \cdot 100.6)=112.63
\end{aligned}
$$

and this does not equal

$$
\begin{aligned}
\mathrm{E}_{Q}\left[\mathrm{E}\left[\widetilde{F C F}_{2} \mid \mathcal{F}_{1}\right] \mid \mathcal{F}_{0}\right]=0.1\left(\frac{1}{2}\right. & \left.\cdot 145.2+\frac{1}{2} \cdot 120\right) \\
& +0.9\left(\frac{1}{2} \cdot 122+\frac{1}{2} \cdot 100.6\right)=113.43
\end{aligned}
$$

In general, it can be shown that both expectations may be changed if downup and up-down yield the same cash flows. Otherwise not. The rule is: If the outcome is independent of the actual path, i.e., if $u d$ yields the same as $d u$ and $u u d$ yields the same as $u d u$ and $d u u$, then expectations may be changed.

3. For the conditional expectation of $\widehat{F C F}_{3}$ given $\mathcal{F}_{2}$ we have four possible realizations: up-up, up-down, down-up, and down-down. We start with up-up and get

$$
\begin{aligned}
\mathrm{E}\left[{\widetilde{F C F_{3}}}_{3} \mid \mathcal{F}_{2}\right](u u) & =\left(u^{3}+u^{2} d\right) F C F_{0} \\
& =u^{2} F C F_{0} \\
& =\widetilde{F C F}_{2}(u u) .
\end{aligned}
$$

The other equations for $u d, d u$ as well as $d d$ follow analogously:

$$
\mathrm{E}\left[\widetilde{F C F}_{3} \mid \mathcal{F}_{2}\right]=\widetilde{F C F}_{2}
$$

4. We have

$$
\mathrm{E}\left[\widetilde{F C F}_{2} \mid \mathcal{F}_{1}\right]= \begin{cases}\frac{u+m+d}{3} \widetilde{F C F}_{1}(u) & \text { if } \widetilde{F C F}_{1} \text { is up, } \\ \frac{u+m+d}{3} \widetilde{F C F}_{1}(m) & \text { if } \widetilde{F C F}_{1} \text { is middle } \\ \frac{u+m+d}{3} \widetilde{F C F}_{1}(d) & \text { if } \widetilde{F C F}_{1} \text { is down. }\end{cases}
$$




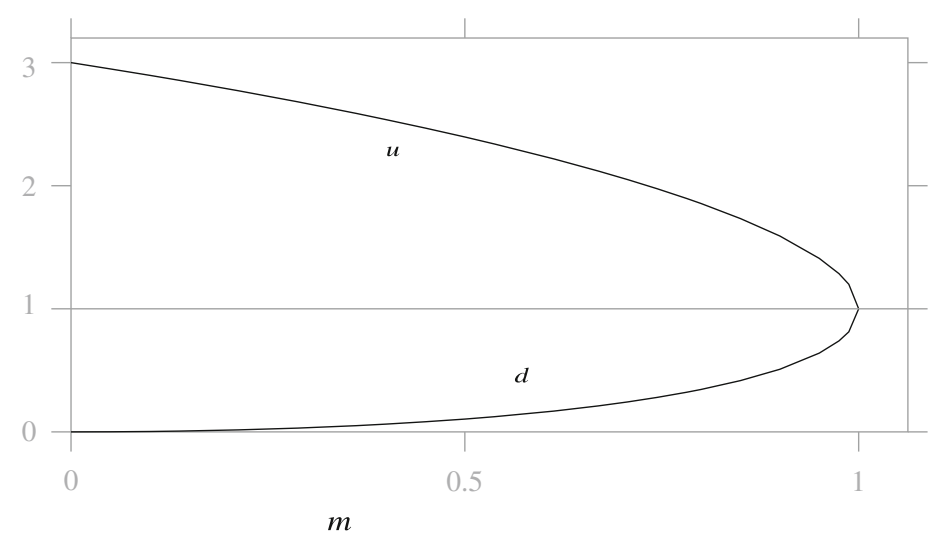

Fig. 7.2 Plot of $(u, m, d)$

The required relation holds if

$$
u+m+d=3 \text {. }
$$

If, for example, $d \in[0,1]$, then this equation jointly with $u d=m^{2}$ gives the solution

$$
d=\frac{1}{2}\left(3-m+\sqrt{3\left(3-2 m-m^{2}\right)}\right), \quad u=\frac{m^{2}}{d} .
$$

Figure 7.2 shows $(u, m, d)$ for $m$ from $[0,1]$.

\subsubsection{A First Glance at Business Values}

1. From $s_{1}-1 \geq s_{2}-1 \geq t$ it follows

$$
\begin{aligned}
\mathrm{E}\left[\widetilde{r}_{s_{1}} \widetilde{r}_{s_{2}} \mid \mathcal{F}_{t}\right] & =\mathrm{E}\left[\mathrm{E}\left[\widetilde{r}_{s_{1}} \widetilde{r}_{s_{2}} \mid \mathcal{F}_{s_{2}}\right] \mid \mathcal{F}_{t}\right] & & \text { by Rule } 4 \\
& =\mathrm{E}\left[\widetilde{r}_{s_{2}} \mathrm{E}\left[\widetilde{r}_{s_{1}} \mid \mathcal{F}_{s_{2}}\right] \mid \mathcal{F}_{t}\right] & & \text { by Rule } 5 \\
& =\mathrm{E}\left[\widetilde{r}_{s_{2}} \mathrm{E}[\underbrace{\mathrm{E}\left[\widetilde{r}_{s_{1}} \mid \mathcal{F}_{s_{1}-1}\right]}_{:=k_{s_{1}}} \mid \mathcal{F}_{s_{2}}] \mid \mathcal{F}_{t}\right] & & \text { by Rule 4. }
\end{aligned}
$$

Now using Definition 2.1 and because $k_{s_{1}}$ is a real number (by assumption) it follows from Rule 2 that

$$
\mathrm{E}\left[\widetilde{r}_{s_{1}} \widetilde{r}_{s_{2}} \mid \mathcal{F}_{t}\right]=k_{s_{1}} \mathrm{E}\left[\widetilde{r}_{s_{2}} \mid \mathcal{F}_{t}\right]
$$


Employing Rule 4 yields

$$
\begin{aligned}
k_{s_{1}} & =\mathrm{E}\left[\mathrm{E}\left[\widetilde{r}_{s_{1}} \mid \mathcal{F}_{s_{1}-1}\right] \mid \mathcal{F}_{t}\right] \\
& =\mathrm{E}\left[\widetilde{r}_{s_{1}} \mid \mathcal{F}_{t}\right]
\end{aligned}
$$

which gives the desired result.

2. $k$ is constant. We have using Theorem 2.1 as well as $(2.5)^{1}$

$$
\begin{aligned}
\widetilde{V}_{t} & =\sum_{s=t+1}^{\infty} \frac{\mathrm{E}\left[\widetilde{F C F}_{s} \mid \mathcal{F}_{t}\right]}{(1+k)^{s-t}} \\
& =\sum_{s=t+1}^{\infty} \frac{(1+g)^{s-t} \widetilde{F C F}_{t}}{(1+k)^{s-t}} \\
& =\frac{1+g}{k-g} \widetilde{F C F}_{t} .
\end{aligned}
$$

Now

$$
\widetilde{V}_{t+1}= \begin{cases}u \widetilde{V}_{t} & \text { if up, } \\ d \widetilde{V}_{t} & \text { if down }\end{cases}
$$

is an immediate consequence of the fact that $\widetilde{F C F}_{t}$ follows this pattern. The second part of the solution follows from $\frac{u}{2}+\frac{d}{2}=0$.

3. We have to show that the firm's value amounts to

$$
V_{0}=\frac{C}{k-g} \text {. }
$$

To prove this statement, we use Theorem 2.1 and know that

$$
\begin{aligned}
V_{0} & =\sum_{t=1}^{\infty} \frac{\mathrm{E}\left[\widetilde{F C F}_{t}\right]}{(1+k)^{t}} \\
& =\sum_{t=1}^{\infty} \frac{(1+g)^{t-1} C}{(1+k)^{t}} \\
& =\frac{C}{1+g} \sum_{t=1}^{\infty}\left(\frac{1+g}{1+k}\right)^{t} \\
& =\frac{C}{k-g} .
\end{aligned}
$$

If $g \geq k$, then the sum does not converge and the value of the firm is infinite.

\footnotetext{
${ }^{1}$ We have not used $g=0$ in our proof.
} 
4. We know that

$$
\widetilde{V}_{t}=\frac{\mathrm{E}_{Q}\left[\widetilde{V}_{t+1}+\widetilde{F C F}_{t+1} \mid \mathcal{F}_{t}\right]}{1+r_{f}}
$$

holds. Now adding $C\left(1+r_{f}\right)^{t}$ to both sides

$$
\widetilde{V}_{t}+C\left(1+r_{f}\right)^{t}=\frac{\mathrm{E}_{Q}\left[\widetilde{V}_{t+1}+C\left(1+r_{f}\right)^{t+1}+\widetilde{F C F}_{t+1} \mid \mathcal{F}_{t}\right]}{1+r_{f}}
$$

and replacing $\widetilde{V}_{t}^{*}:=\widetilde{V}_{t}+C\left(1+r_{f}\right)^{t}$ gives the desired result

$$
\widetilde{V}_{t}^{*}=\frac{\mathrm{E}_{Q}\left[\widetilde{V}_{t+1}^{*}+\widetilde{F C F}_{t+1} \mid \mathcal{F}_{t}\right]}{1+r_{f}}
$$

Both $\widetilde{V}$ as well as $\widetilde{V}^{*}$ satisfy the fundamental theorem.

Note that $\widetilde{V}^{*}$ does not satisfy the transversality condition. We have

$$
\lim _{T \rightarrow \infty} \frac{\mathrm{E}_{Q}\left[\widetilde{V}_{T}+C\left(1+r_{f}\right)^{T} \mid \mathcal{F}_{t}\right]}{\left(1+r_{f}\right)^{T-t}}=\lim _{T \rightarrow \infty} \frac{\mathrm{E}_{Q}\left[\widetilde{V}_{T} \mid \mathcal{F}_{t}\right]}{\left(1+r_{f}\right)^{T-t}}+C\left(1+r_{f}\right)^{t}
$$

and this cannot be zero for all $C$.

5. By plugging in the assumption into the expectation under $Q$ we get

$$
\begin{aligned}
\frac{\mathrm{E}_{Q}\left[\widetilde{V}_{t+1}+\widetilde{F C F}_{t+1} \mid \mathcal{F}_{t}\right]}{1+r_{f}} & =\frac{\mathrm{E}_{Q}\left[\frac{\widetilde{F C F}_{0}+g}{r_{f}}+{\widetilde{F C F_{0}}}+\varepsilon_{t+1} \mid \mathcal{F}_{t}\right]}{1+r_{f}} \\
& =\frac{\frac{{\widetilde{F C F_{0}}}_{r_{f}}}{1+r_{f}}+\widetilde{F C}_{0}+g}{1+\widetilde{F}_{f}} \\
& =\frac{1}{r_{f}\left(1+r_{f}\right)}\left(\widetilde{F C F}_{0}+g+\left(\overline{F C F_{0}}+g\right) r_{f}\right) \\
& =\frac{\widetilde{F C F_{0}}+g}{r_{f}}=\widetilde{V}_{t}
\end{aligned}
$$

which is the fundamental theorem. 
Also, transversality is easily checked.

$$
\begin{aligned}
\lim _{T \rightarrow \infty} \frac{\mathrm{E}_{Q}\left[\widetilde{V}_{T} \mid \mathcal{F}_{t}\right]}{\left(1+r_{f}\right)^{T-t}} & =\lim _{T \rightarrow \infty} \frac{\mathrm{E}_{Q}\left[\frac{{\widetilde{F C F_{0}}}_{r_{f}}}{r_{t}} \mid \mathcal{F}_{t}\right]}{\left(1+r_{f}\right)^{T-t}} \\
& =\lim _{T \rightarrow \infty} \frac{\widetilde{F C F_{0}}+g}{r_{f}\left(1+r_{f}\right)^{T-t}}=0
\end{aligned}
$$

because $r_{f}>0$.

\subsection{Corporate Income Tax}

\subsubsection{Unlevered Firms}

1. (a) First, if a random variable $\varepsilon_{t}$ can have two values we speak of a binomial structure. In order to get a typical binomial tree from

$$
\widetilde{F C F}_{t}^{u}=1+\varepsilon_{1}+\varepsilon_{2}+\ldots+\varepsilon_{t}
$$

we have to verify that for any cash flow not the actual movement but only the number of up's and down's is decisive. In other words, "uudd" as well as "uddu," etc. must lead to the same cash flow. But this is evident from the fact that the increments are added.

(b) For the logarithmized cash flows

$$
\log \left(\widetilde{F C F}_{t}^{u}\right)=\log \left(1+\varepsilon_{1}\right)+\log \left(1+\varepsilon_{2}\right) \cdots \log \left(1+\varepsilon_{t}\right) .
$$

Any $\log \left(1+\varepsilon_{t}\right)$ is binomial as in 1a. Hence, the proposition holds.

2. (a) This follows immediately from the fact that the sum of two standard normally distributed variables is normally distributed again, the variance being the sum of the individual variances.

(b) Following the above solution we have

$$
\log \left(\widetilde{F C F}_{t}^{u}\right)=\log \left(1+\varepsilon_{1}\right)+\log \left(1+\varepsilon_{2}\right) \cdots \log \left(1+\varepsilon_{t}\right) .
$$

Any summand on the right-hand side is normally distributed with expectation 0 and variance 1 . The sum is therefore normally distributed with variance $t$ and the claim is proven.

3. We have

$$
\begin{aligned}
\mathrm{E}\left[\widetilde{F C F}_{t+1}^{u} \mid \mathcal{F}_{t}\right] & =\mathrm{E}\left[\left(1+g_{t}\right) \widetilde{F C F}_{t}^{u}+\varepsilon_{t+1} \mid \mathcal{F}_{t}\right] \\
& =\left(1+g_{t}\right) \widetilde{F C F}_{t}^{u}
\end{aligned}
$$


by Rule 5. Furthermore, for $s>t$

$$
\begin{array}{rlrl}
\operatorname{Cov}\left[\varepsilon_{s}, \varepsilon_{t}\right] & =\mathrm{E}\left[\varepsilon_{s} \varepsilon_{t}\right] & \\
& =\mathrm{E}\left[\mathrm{E}\left[\varepsilon_{s} \varepsilon_{t} \mid \mathcal{F}_{t}\right]\right] & & \text { by Rule } 4 \\
& =\mathrm{E}\left[\varepsilon_{t} \mathrm{E}\left[\varepsilon_{s} \mid \mathcal{F}_{t}\right]\right] & & \text { by Rule } 5 \\
& =\mathrm{E}\left[\varepsilon_{t} \mathrm{E}[\underbrace{\mathrm{E}\left[\varepsilon_{s} \mid \mathcal{F}_{s-1}\right]}_{=0} \mid \mathcal{F}_{t}]\right] & & \text { by Rule } 4 \\
& =0 . &
\end{array}
$$

4. (a) From Fig. 3.2 we get for the noise term

$$
\varepsilon_{2}= \begin{cases}\varepsilon(u u) & \text { if development is } u u, \\ \varepsilon(u d) & \text { if development is } u d, \\ \varepsilon(d u) & \text { if development is } d u \\ \varepsilon(d d) & \text { if development is } d d .\end{cases}
$$

The first equation of the problem requires

$$
0=\mathrm{E}\left[\varepsilon_{2}\right]=\mathrm{E}\left[\varepsilon_{2} \mid \mathcal{F}_{1}\right]
$$

or

$$
0=\frac{\varepsilon(u u)+\varepsilon(u d)+\varepsilon(d u)+\varepsilon(d d)}{4}= \begin{cases}\frac{\varepsilon(u u)+\varepsilon(u d)}{2} & \text { if } u, \\ \frac{\varepsilon(d u)+\varepsilon(d d)}{2} & \text { if } d .\end{cases}
$$

Hence, we have

$$
\frac{\varepsilon(u u)+\varepsilon(u d)}{2}=\frac{\varepsilon(d u)+\varepsilon(d d)}{2}=0 .
$$

This is the only requirement that has to be satisfied for the noise terms. A parametrized solution is

$$
\varepsilon=\left\{\begin{array}{cl}
x & \text { if } u u \\
-x & \text { if } u d \\
y & \text { if } d u \\
-y & \text { if } d d
\end{array}\right.
$$

for arbitrary $x, y$. 
(b) The second equation of the problem

$$
\mathrm{E}\left[f\left(\varepsilon_{2}\right)\right]=\mathrm{E}\left[f\left(\varepsilon_{2}\right) \mid \mathcal{F}_{1}\right]
$$

is equivalent to

$$
\frac{f(\varepsilon(u u))+f(\varepsilon(u d))+f(\varepsilon(d u))+f(\varepsilon(d d))}{4}= \begin{cases}\frac{f(\varepsilon(u u))+f(\varepsilon(u d))}{2} & \text { if } u, \\ \frac{f(\varepsilon(d u))+f(\varepsilon(d d))}{2} & \text { if } d .\end{cases}
$$

Since $f$ is an arbitrary function, it is straightforward to see that a sufficient and necessary condition is given by the following cases ${ }^{2}$ :

Case 1: The following equations have to be satisfied:

$$
\varepsilon(u u)=\varepsilon(d u), \quad \text { and } \quad \varepsilon(u d)=\varepsilon(d d) .
$$

Case 2: The following equations have to be satisfied:

$$
\varepsilon(u u)=\varepsilon(d d), \quad \text { and } \quad \varepsilon(u d)=\varepsilon(d u) .
$$

We will from now on only consider case 1.

Since the increments must have expectation zero, we furthermore have to add the requirement

$$
\varepsilon(u u)+\varepsilon(u d)=0, \quad \text { and } \quad \varepsilon(d u)+\varepsilon(d d)=0 .
$$

Hence, there is only one possible solution

$$
\varepsilon=\left\{\begin{array}{cl}
x & \text { if } u u \\
-x & \text { if } u d \\
x & \text { if } d u \\
-x & \text { if } d d
\end{array}\right.
$$

${ }^{2}$ The formal argument is: if

$$
\frac{f(w)+f(x)+f(y)+f(z)}{4}=\frac{f(w)+f(x)}{2}=\frac{f(y)+f(z)}{2}
$$

has to hold regardless of what $f$ is, we must have $w=y$ and $x=z$ or $w=z$ and $x=y$. Otherwise, we could change the value $f(w)$ without changing the function $f$ outside $w$ and get a violation of the above assumption. 
for arbitrary $x$. The second case gives us

$$
\varepsilon=\left\{\begin{array}{cl}
x & \text { if } u u, \\
-x & \text { if } u d, \\
-x & \text { if } d u, \\
x & \text { if } d d,
\end{array}\right.
$$

This is far more restrictive than the linear relation from $4 \mathrm{a}$.

Remark The two conditions "independent increments" and " $\widetilde{F C F}_{2}^{u}(u d)=$ $\widetilde{F C F}_{2}^{u}(d u)$ " require $\widetilde{F C F}_{1}^{u}$ to be chosen properly: The two conditions imply

$$
\widetilde{F C F}_{1}^{u}(u)+\varepsilon(u d)=\widetilde{F C F}_{1}^{u}(d)+\varepsilon(d u)
$$

and with independent increments and $\widetilde{F C F}_{1}^{u}(u)=133.1$ as well as $\widetilde{F C F}_{1}^{u}(d)=110.8$ this cannot hold.

5. The solution of this problem runs absolutely parallel to the solution of problem 4 . Noise terms are zero if and only if

$$
\frac{\varepsilon(u u)+\varepsilon(u d)}{2}=\frac{\varepsilon(d u)+\varepsilon(d d)}{2}=0 .
$$

A parametrized solution is

$$
\varepsilon=\left\{\begin{array}{cl}
x & \text { if } u u, \\
-x & \text { if } u d, \\
y & \text { if } d u, \\
-y & \text { if } d d
\end{array}\right.
$$

for arbitrary $x, y$.

Noise terms are furthermore independent if

$$
\varepsilon=\left\{\begin{array}{cl}
x & \text { if } u u, \\
-x & \text { if } u d, \\
x & \text { if } d u, \\
-x & \text { if } d d,
\end{array}\right.
$$


or

$$
\varepsilon=\left\{\begin{aligned}
x & \text { if } u u \\
-x & \text { if } u d \\
-x & \text { if } d u \\
x & \text { if } d d
\end{aligned}\right.
$$

for arbitrary $x$.

This solution for $\varepsilon$ is different from problem 4 . In problem 4 the (noise) increments were additive. However, here they are multiplicative.

6. We have

$$
\begin{aligned}
\operatorname{div}_{t} & =\frac{\mathrm{E}\left[\widetilde{F C F}_{t+1}^{u} \mid \mathcal{F}_{t}\right]}{\widetilde{V}_{t}^{u}} \\
& =\frac{\left(1+g_{t}\right) \widetilde{F C F}_{t}^{u}}{\widetilde{V}_{t}^{u}}=\left(1+g_{t}\right) d_{t}^{u}
\end{aligned}
$$

and

$$
\begin{aligned}
\operatorname{gain}_{t}= & \frac{\mathrm{E}\left[\widetilde{V}_{t+1}^{u}-\widetilde{V}_{t}^{u} \mid \mathcal{F}_{t}\right]}{\widetilde{V}_{t}^{u}} \\
= & \frac{\mathrm{E}\left[\frac{\widetilde{F C F}_{t+1}^{u}}{d_{t+1}^{u}}-\frac{\widetilde{F C F}_{t}^{u}}{d_{t}^{u}} \mid \mathcal{F}_{t}\right]}{\widetilde{F C F}_{t}^{u}} \\
= & \frac{\frac{1+g_{t}^{u}}{d_{t+1}^{u}} \widetilde{F C F}_{t}^{u}-\frac{\widetilde{F C F}_{t}^{u}}{\widetilde{F C F}_{t}^{u}}}{d_{t}^{u}} \\
= & \left(1+g_{t}\right) \frac{d_{t}^{u}}{d_{t+1}^{u}}-1 .
\end{aligned}
$$

7. (a) In this case

$$
\begin{aligned}
\widetilde{V}_{t}^{u} & =\sum_{s=t+1}^{\infty} \frac{\mathrm{E}\left[\widetilde{F C F}_{s}^{u} \mid \mathcal{F}_{t}\right]}{\left(1+k^{E, u}\right)^{s-t}} \\
& =\sum_{s=t+1}^{\infty} \frac{(1+g)^{s-t} \widetilde{F C F}_{t}^{u}}{\left(1+k^{E, u}\right)^{s-t}}
\end{aligned}
$$




$$
\begin{aligned}
& =\widetilde{F C F}_{t}^{u} \sum_{s=t+1}^{\infty}\left(\frac{1+g}{1+k^{E, u}}\right)^{s-t} \\
& =\widetilde{F C F}_{t}^{u} \frac{1+g}{k^{E, u}-g}
\end{aligned}
$$

if $g<k^{E, u}$.

The dividend-price ratio is $\frac{k^{E, u}-g}{1+g}$.

Using this relation the dividend ratio is

$$
\begin{aligned}
\operatorname{div}_{t} & =\frac{\mathrm{E}\left[\widetilde{F C F}_{t+1}^{u} \mid \mathcal{F}_{t}\right]}{\widetilde{V}_{t}^{u}} \\
& =\frac{(1+g) \widetilde{F C F}_{t}^{u}}{\widetilde{F C F}_{t}^{u}} \frac{k^{E, u}-g}{1+g} \\
& =k^{E, u}-g
\end{aligned}
$$

and the capital gains ratio is

$$
\begin{aligned}
\operatorname{gain}_{t} & =\frac{\mathrm{E}\left[\widetilde{V}_{t+1}^{u}-\widetilde{V}_{t}^{u} \mid \mathcal{F}_{t}\right]}{\widetilde{V}_{t}^{u}} \\
& =\frac{\mathrm{E}\left[\frac{(1+g) \widetilde{F C F}_{t+1}^{u}}{k^{E, u}-g}-\frac{(1+g) \widetilde{F C F}_{t}^{u}}{k^{E, u}-g} \mid \mathcal{F}_{t}\right]}{\frac{(1+g) \widetilde{F C F}_{t}^{u}}{k^{E, u}-g}} \\
& =\frac{g \widetilde{F C F}_{t}^{u}}{\widetilde{F C F}_{t}^{u}} \\
& =g .
\end{aligned}
$$

(b) In this case the firm value is infinite.

(c) From the fundamental theorem we have

$$
\begin{aligned}
\widetilde{V}_{t}^{u} & =\frac{\mathrm{E}_{Q}\left[\widetilde{F C F}_{t+1}^{u}+\widetilde{V}_{t+1}^{u} \mid \mathcal{F}_{t}\right]}{1+r_{f}} \\
\widetilde{F C F}_{t}^{u} \frac{1+g}{k^{E, u}-g}= & \frac{\mathrm{E}_{Q}\left[\widetilde{F C F}_{t+1}^{u}+\widetilde{F C F}_{t+1}^{u} \frac{1+g}{k^{E, u}-g} \mid \mathcal{F}_{t}\right]}{1+r_{f}} \\
\mathrm{E}_{Q}\left[\widetilde{F C F}_{t+1}^{u} \mid \mathcal{F}_{t}\right] & =\frac{(1+g)\left(1+r_{f}\right)}{1+k^{E, u}} \widetilde{F C F}_{t}^{u} .
\end{aligned}
$$


8. (a) First we have by Rule 4

$$
\begin{aligned}
\mathrm{E}\left[\widetilde{F C F}_{t+2}^{u} \mid \mathcal{F}_{t}\right] & =\mathrm{E}\left[\mathrm{E}\left[\widetilde{F C F}_{t+2}^{u} \mid \mathcal{F}_{t+1}\right] \mid \mathcal{F}_{t}\right] \\
& =\mathrm{E}\left[\widetilde{F C F}_{t+1}^{u}+X_{t+1} \mid \mathcal{F}_{t}\right] \\
& =\mathrm{E}\left[\widetilde{F C F}_{t+1}^{u} \mid \mathcal{F}_{t}\right] \\
& =\widetilde{F C F}_{t}^{u}+X_{t}
\end{aligned}
$$

and hence for $s>t$

$$
\mathrm{E}\left[\widetilde{F C F}_{s}^{u} \mid \mathcal{F}_{t}\right]=\widetilde{F C F}_{t}^{u}+X_{t}
$$

The value of the company is given by

$$
\begin{aligned}
\widetilde{V}_{t}^{u} & =\sum_{s=t+1}^{\infty} \frac{\mathrm{E}\left[\widetilde{F C F}_{s}^{u} \mid \mathcal{F}_{t}\right]}{\left(1+k^{E, u}\right)^{s-t}} \\
& =\sum_{s=t+1}^{\infty} \frac{\widetilde{F C F}_{t}^{u}+X_{t}}{\left(1+k^{E, u}\right)^{s-t}} \\
& =\frac{\widetilde{F C F}_{t}^{u}}{k^{E, u}}+\frac{X_{t}}{k^{E, u}} .
\end{aligned}
$$

Since $\widetilde{F C F}_{t}^{u}$ and $X_{t}$ are uncorrelated the variance of the firm is greater than the variance of the cash flows (if $k^{E, u}<100 \%$ ),

$$
\begin{aligned}
\operatorname{Var}\left[\widetilde{V}_{t}^{u}\right] & =\operatorname{Var}\left[\frac{\widetilde{F C F}_{t}^{u}}{k^{E, u}}+\frac{X_{t}}{k^{E, u}}\right] \\
& =\frac{\operatorname{Var}\left[\widetilde{F C F}_{t}^{u}\right]}{\left(k^{E, u}\right)^{2}}+\frac{\operatorname{Var}\left[X_{t}\right]}{\left(k^{E, u}\right)^{2}} \\
& >\frac{\operatorname{Var}\left[\widetilde{F C F}_{t}^{u}\right]}{\left(k^{E, u}\right)^{2}}>\operatorname{Var}\left[\widetilde{F C F}_{t}^{u}\right] .
\end{aligned}
$$

(b) Now

$$
\mathrm{E}\left[\widetilde{V}_{t+1}^{u} \mid \mathcal{F}_{t}\right]=\mathrm{E}\left[\frac{\widetilde{F C F}_{t+1}^{u}}{k^{E, u}}+\frac{X_{t+1}}{k^{E, u}} \mid \mathcal{F}_{t}\right]
$$




$$
\begin{aligned}
& =\frac{\mathrm{E}\left[\widetilde{F C F}_{t+1}^{u} \mid \mathcal{F}_{t}\right]}{k^{E, u}} \\
& =\frac{\widetilde{F C F}_{t}^{u}+X_{t}}{k^{E, u}}=\widetilde{V}_{t}^{u}
\end{aligned}
$$

and the expected capital gains rate is zero.

(c) This is harder to show and we closely follow the proof of Theorem 3.3.

$$
\begin{aligned}
\frac{\mathrm{E}\left[\widetilde{F C F}_{t+1}^{u}+\widetilde{V}_{t+1}^{u} \mid \mathcal{F}_{t}\right]}{1+k^{E, u}} & =\frac{\mathrm{E}_{Q}\left[\widetilde{F C F}_{t+1}^{u}+\widetilde{V}_{t+1}^{u} \mid \mathcal{F}_{t}\right]}{1+r_{f}}=\widetilde{V}_{t}^{u} \\
\frac{\mathrm{E}\left[\widetilde{F C F}_{t+1}^{u}+\frac{\left.\widetilde{F C F}_{t+1}^{u}+X_{t+1} \mid \mathcal{F}_{t}\right]}{k^{E, u}}\right.}{1+k^{E, u}} & =\frac{\mathrm{E}_{Q}\left[\widetilde{F C F}_{t+1}^{u}+\frac{\widetilde{F C F}_{t+1}^{u}+X_{t+1}}{k^{E, u}} \mid \mathcal{F}_{t}\right]}{1+r_{f}} \\
\frac{\left(1+\frac{1}{k^{E, u}}\right) \mathrm{E}\left[\widetilde{F C F}_{t+1}^{u} \mid \mathcal{F}_{t}\right]}{1+k^{E, u}} & =\frac{\left(1+\frac{1}{k^{E, u}}\right) \mathrm{E}_{Q}\left[\widetilde{F C F}_{t+1}^{u} \mid \mathcal{F}_{t}\right]}{1+r_{f}} \\
\frac{\mathrm{E}\left[\widetilde{F C F}_{t+1}^{u} \mid \mathcal{F}_{t}\right]}{1+k^{E, u}} & =\frac{\mathrm{E}_{Q}\left[\widetilde{F C F}_{t+1}^{u} \mid \mathcal{F}_{t}\right]}{1+r_{f}}
\end{aligned}
$$

which is the first part of the claim.

The second part follows from

$$
\begin{aligned}
\frac{\mathrm{E}\left[\widetilde{F C F}_{t+1}^{u}+\widetilde{V}_{t+1}^{u} \mid \mathcal{F}_{t}\right]}{1+k^{E, u}} & =\frac{\mathrm{E}_{Q}\left[\widetilde{F C F}_{t+1}^{u}+\widetilde{V}_{t+1}^{u} \mid \mathcal{F}_{t}\right]}{1+r_{f}}=\widetilde{V}_{t}^{u} \\
\frac{\mathrm{E}\left[\widetilde{V}_{t+1}^{u} \mid \mathcal{F}_{t}\right]}{1+k^{E, u}} & =\frac{\mathrm{E}_{Q}\left[\widetilde{V}_{t+1}^{u} \mid \mathcal{F}_{t}\right]}{1+r_{f}} \\
\frac{\mathrm{E}\left[\frac{\mathrm{E}\left[\widetilde{F C F}_{t+2}^{u}+\widetilde{V}_{t+2}^{u} \mid \mathcal{F}_{t+1}\right]}{1+k^{E, u}} \mid \mathcal{F}_{t}\right]}{1+k^{E, u}} & =\frac{\mathrm{E}_{Q}\left[\frac{\mathrm{E}_{Q}\left[\widetilde{F C F}_{t+2}^{u}+\widetilde{V}_{t+2}^{u} \mid \mathcal{F}_{t+1}\right]}{1+r_{f}} \mid \mathcal{F}_{t}\right]}{1+r_{f}} \\
\frac{\mathrm{E}\left[\widetilde{F C F}_{t+2}^{u}+\widetilde{V}_{t+2}^{u} \mid \mathcal{F}_{t}\right]}{\left(1+k^{E, u}\right)^{2}} & =\frac{\mathrm{E}_{Q}\left[\widetilde{F C F}_{t+2}^{u}+\widetilde{V}_{t+2}^{u} \mid \mathcal{F}_{t}\right]}{\left(1+r_{f}\right)^{2}} \\
\frac{\mathrm{E}\left[\widetilde{F C F}_{t+2}^{u}+\frac{\widetilde{F C F}_{t+2}^{u}+X_{t+2}}{k^{E, u}} \mid \mathcal{F}_{t}\right]}{\left(1+k^{E, u}\right)^{2}} & =\frac{\mathrm{E}_{Q}\left[\widetilde{F C F}_{t+2}^{u}+\frac{\widetilde{F C F}_{t+2}^{u}+X_{t+2}}{k^{E, u}} \mid \mathcal{F}_{t}\right]}{\left(1+r_{f}\right)^{2}}
\end{aligned}
$$


and since $\mathrm{E}\left[X_{t+2} \mid \mathcal{F}_{t+1}\right]=\mathrm{E}_{Q}\left[X_{t+2} \mid \mathcal{F}_{t+1}\right]=0$,

$$
\frac{\left(1+\frac{1}{k^{E, u}}\right) \mathrm{E}\left[\widetilde{F C F}_{t+2}^{u} \mid \mathcal{F}_{t}\right]}{\left(1+k^{E, u}\right)^{2}}=\frac{\left(1+\frac{1}{k^{E, u}}\right) \mathrm{E}_{Q}\left[\widetilde{F C F}_{t+2}^{u} \mid \mathcal{F}_{t}\right]}{\left(1+r_{f}\right)^{2}}
$$

which is almost the claim.

9. (a) First we have

$$
\mathrm{E}\left[\widetilde{F C F}_{s}^{u} \mid \mathcal{F}_{t}\right]=(s-t) C+\widetilde{F C F}_{t}^{u}
$$

by induction. But then

$$
\begin{aligned}
\widetilde{V}_{t}^{u} & =\sum_{s=t+1}^{\infty} \frac{\mathrm{E}\left[\widetilde{F C F}_{s}^{u} \mid \mathcal{F}_{t}\right]}{\left(1+k^{E, u}\right)^{s-t}} \\
& =\sum_{s=t+1}^{\infty} \frac{{\widetilde{F C F_{t}^{u}}}^{u}(s-t) C}{\left(1+k^{E, u}\right)^{s-t}} \\
& =\sum_{s=t+1}^{\infty} \frac{\widetilde{F C F}_{t}^{u}}{\left(1+k^{E, u}\right)^{s-t}}+\sum_{s=t+1}^{\infty} \frac{(s-t) C}{\left(1+k^{E, u}\right)^{s-t}} \\
& =\frac{\widetilde{F C F}_{t}^{u}}{k^{E, u}}+\frac{1+k^{E, u}}{\left(k^{E, u}\right)^{2}} C
\end{aligned}
$$

using an algebraic formula. ${ }^{3}$ In particular, the price-dividend ratio is not deterministic any more. Compare this to problem 3.

${ }^{3}$ Note that

$$
\sum_{s=1}^{\infty} \frac{1}{(1+x)^{s}}=\frac{1}{x}
$$

Differentiating this relation yields

$$
\sum_{s=1}^{\infty} \frac{-s}{(1+x)^{s+1}}=-\frac{1}{x^{2}}
$$

or after multiplying by $-(1+x)$

$$
\sum_{s=1}^{\infty} \frac{s}{(1+x)^{s}}=\frac{1+x}{x^{2}}
$$


(b) We have

$$
\begin{aligned}
\mathrm{E}\left[\widetilde{V}_{t+1}^{u} \mid \mathcal{F}_{t}\right] & =\mathrm{E}\left[\frac{\widetilde{F C F}_{t+1}^{u}}{k^{E, u}}+\frac{1+k^{E, u}}{\left(k^{E, u}\right)^{2}} C \mid \mathcal{F}_{t}\right] \\
& =\frac{\widetilde{F C F}_{t}^{u}+C}{k^{E, u}}+\frac{1+k^{E, u}}{\left(k^{E, u}\right)^{2}} C \\
& =\widetilde{V}_{t}^{u}+\frac{C}{k^{E, u}}
\end{aligned}
$$

and hence the expected capital gains rate is not zero.

(c) We use

$$
\frac{\mathrm{E}\left[\widetilde{F C F}_{t+1}^{u}+\widetilde{V}_{t+1}^{u} \mid \mathcal{F}_{t}\right]}{1+k^{E, u}}=\frac{\mathrm{E}_{Q}\left[\widetilde{F C F}_{t+1}^{u}+\widetilde{V}_{t+1}^{u} \mid \mathcal{F}_{t}\right]}{1+r_{f}}=\widetilde{V}_{t}^{u}
$$

Employing the above result this implies

$$
\frac{\mathrm{E}\left[\widetilde{F C F}_{t+1}^{u}+\frac{\widetilde{F C F}_{t+1}^{u}}{k^{E, u}}+\frac{1+k^{E, u}}{\left(k^{E, u}\right)^{2}} C \mid \mathcal{F}_{t}\right]}{1+k^{E, u}}=\frac{\mathrm{E}_{Q}\left[\widetilde{F C F}_{t+1}^{u}+\frac{\widetilde{F C F}_{t+1}^{u}}{k^{E, u}}+\frac{1+k^{E, u}}{\left(k^{E, u}\right)^{2}} C \mid \mathcal{F}_{t}\right]}{1+r_{f}}
$$

and after rearranging and multiplying by $\frac{k^{E, u}}{1+k^{E, u}}$,

$$
\frac{\mathrm{E}\left[\widetilde{F C F}_{t+1}^{u} \mid \mathcal{F}_{t}\right]+\frac{1}{k^{E, u}} C}{1+k^{E, u}}=\frac{\mathrm{E}_{Q}\left[\widetilde{F C F}_{t+1}^{u} \mid \mathcal{F}_{t}\right]+\frac{1}{k^{E, u}} C}{1+r_{f}}
$$

Rearranging once again gives

$$
\mathrm{E}_{Q}\left[\widetilde{F C F}_{t+1}^{u} \mid \mathcal{F}_{t}\right]=\frac{1+r_{f}}{1+k^{E, u}} \mathrm{E}\left[\widetilde{F C F}_{t+1}^{u} \mid \mathcal{F}_{t}\right]+\frac{1+r_{f}}{k^{E, u}}\left(\frac{1}{1+k^{E, u}}-\frac{1}{1+r_{f}}\right) C
$$

which is, after using the assumption,

$$
\mathrm{E}_{Q}\left[\widetilde{F C F}_{t+1}^{u} \mid \mathcal{F}_{t}\right]=\frac{1+r_{f}}{1+k^{E, u}}\left(\widetilde{F C F}_{t}^{u}+C\right)+\frac{C}{k^{E, u}}\left(\frac{1+r_{f}}{1+k^{E, u}}-1\right) .
$$

Summarizing the terms with $C$ gives the required result.

Now consider Theorem 3.3. Since $C$ does not cancel in (7.1) this intermediate result shows that our weak auto-regressive assumption will violate Theorem 3.3. We have

$$
\frac{C}{k^{E, u}\left(1+k^{E, u}\right)} \neq \frac{C}{k^{E, u}\left(1+r_{f}\right)} \Longrightarrow \frac{\mathrm{E}\left[\overline{F C F}_{t+1}^{u} \mid \mathcal{F}_{t}\right]}{1+k^{E, u}} \neq \frac{\mathrm{E}_{Q}\left[\widetilde{F C F}_{t+1}^{u} \mid \mathcal{F}_{t}\right]}{1+r_{f}}
$$


Hence, using this assumption about cash flows we can still use cost of capital as discount rates but we have to add a correction term due to the fact that $C \neq 0$.

10. (a) From the definition of discount rates (Definition 3.2) we have

$$
\frac{\mathrm{E}_{Q}\left[\widehat{F C F}_{t+2}^{u} \mid \mathcal{F}_{t}\right]}{\left(1+r_{f}\right)^{2}}=\frac{\mathrm{E}\left[\widehat{F C F}_{t+2}^{u} \mid \mathcal{F}_{t}\right]}{\left(1+\kappa_{t}\right)\left(1+\kappa_{t+1}\right)}
$$

From Rule 4 it follows that

$$
\frac{1}{1+r_{f}} \frac{\mathrm{E}_{Q}\left[\mathrm{E}_{Q}\left[\widehat{F C F}_{t+2}^{u} \mid \mathcal{F}_{t+1}\right] \mid \mathcal{F}_{t}\right]}{1+r_{f}}=\frac{\mathrm{E}\left[\mathrm{E}\left[\widetilde{F C F}_{t+2}^{u} \mid \mathcal{F}_{t+1}\right] \mid \mathcal{F}_{t}\right]}{\left(1+\kappa_{t}\right)\left(1+\kappa_{t+1}\right)} .
$$

Independence and (2.5) implies that the outer expectation can be neglected,

$$
\frac{1}{1+r_{f}} \frac{\mathrm{E}_{Q}\left[\widetilde{F C F}_{t+2}^{u} \mid \mathcal{F}_{t+1}\right]}{1+r_{f}}=\frac{1}{1+\kappa_{t}} \frac{\mathrm{E}\left[\widetilde{F C F}_{t+2}^{u} \mid \mathcal{F}_{t+1}\right]}{1+\kappa_{t+1}} .
$$

The term on the right-hand side is by definition of a discount rate equal to

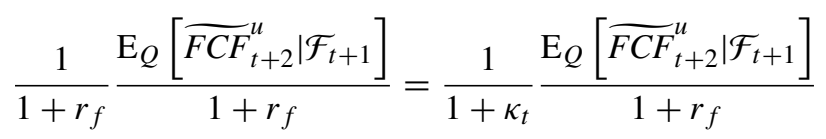

implying $r_{f}=\kappa_{t}$. This argument will apply to all $\kappa_{t}$.

(b) If all discount rates $\kappa_{t}$ are equal to the riskless rate, then the firm value is given by

$$
V_{0}=\sum_{t=1}^{T} \frac{\mathrm{E}\left[{\widetilde{F C F_{t}}}_{t}\right.}{\left(1+\kappa_{1}\right) \ldots\left(1+\kappa_{t}\right)}=\sum_{t=1}^{T} \frac{\mathrm{E}\left[\widetilde{F C F}_{t}\right]}{\left(1+r_{f}\right)^{t}}
$$

and this obviously violates the fact that $\mathrm{E}\left[\widetilde{F C F}_{t}\right] \neq \mathrm{E}_{Q}\left[\widetilde{F C F}_{t}\right]$ and the fundamental theorem

$$
V_{0}=\sum_{t=1}^{T} \frac{\mathrm{E}_{Q}\left[\widetilde{F C F}_{t}\right]}{\left(1+r_{f}\right)^{t}}
$$

There is no other conclusion to be drawn from this problem than that in this case the definition of discount rate $\kappa_{t}^{r \rightarrow s}$ cannot be simplified by omitting $r$ and $s$ from $\kappa_{t}$. It is necessary to specify the time $s$ when the cash flow is paid and the time $r$ when the cash flow is valued as well. 


\subsubsection{Basics About Levered Firms}

1. We start with (3.19) and get for $s>t$

$$
\mathrm{E}_{Q}\left[\widetilde{D}_{s} \mid \mathcal{F}_{t}\right]=\frac{\mathrm{E}_{Q}\left[\widetilde{I}_{s+1}+\widetilde{D}_{s+1}+\widetilde{R}_{s+1} \mid \mathcal{F}_{t}\right]}{1+r_{f}} .
$$

At time $s+1$ we similarly have

$$
\mathrm{E}_{Q}\left[\widetilde{D}_{s+1} \mid \mathcal{F}_{t}\right]=\frac{\mathrm{E}_{Q}\left[\widetilde{I}_{s+2}+\widetilde{D}_{s+2}+\widetilde{R}_{s+2} \mid \mathcal{F}_{t}\right]}{1+r_{f}} .
$$

Plugging this into the first equation gives

$$
\begin{aligned}
\mathrm{E}_{Q}\left[\widetilde{D}_{s} \mid \mathcal{F}_{t}\right] & =\frac{\mathrm{E}_{Q}\left[\widetilde{I}_{s+1}+\frac{\mathrm{E}_{Q}\left[\widetilde{I}_{s+2}+\widetilde{D}_{s+2}+\widetilde{R}_{s+2} \mid \mathcal{F}_{t}\right]}{1+r_{f}}+\widetilde{R}_{s+1} \mid \mathcal{F}_{t}\right]}{1+r_{f}} \\
& =\frac{\mathrm{E}_{Q}\left[\widetilde{I}_{s+1}+\widetilde{R}_{s+1} \mid \mathcal{F}_{t}\right]}{1+r_{f}}+\frac{\mathrm{E}_{Q}\left[\widetilde{I}_{s+2}+\widetilde{R}_{s+2} \mid \mathcal{F}_{t}\right]}{\left(1+r_{f}\right)^{2}}+\frac{\mathrm{E}_{Q}\left[\widetilde{D}_{s+2} \mid \mathcal{F}_{t}\right]}{\left(1+r_{f}\right)^{2}}
\end{aligned}
$$

Continuing this approach we get for $s=t$

$$
\mathrm{E}_{Q}\left[\widetilde{D}_{t} \mid \mathcal{F}_{t}\right]=\sum_{s=t+1}^{T} \frac{\mathrm{E}_{Q}\left[\widetilde{I}_{s}+\widetilde{R}_{s} \mid \mathcal{F}_{t}\right]}{\left(1+r_{f}\right)^{s-t}}+\frac{\mathrm{E}_{Q}\left[\widetilde{D}_{T} \mid \mathcal{F}_{t}\right]}{\left(1+r_{f}\right)^{T-t}}
$$

Financing is always such that $\widetilde{D}_{T}=0$ and $D_{t}=\mathrm{E}_{Q}\left[\widetilde{D}_{t} \mid \mathcal{F}_{t}\right]$ (Rule 5). This proves the claim.

2. The tax shield satisfies

$$
\widetilde{V}_{0}^{l}-\widetilde{V}_{0}^{u}=\sum_{t=0}^{2} \frac{\tau r_{f} \mathrm{E}_{Q}\left[\widetilde{D}_{t}\right]}{\left(1+r_{f}\right)^{t}}
$$

Hence, we have to evaluate the expectations $\mathrm{E}_{Q}\left[\widetilde{D}_{t}\right]$. We get

$$
\mathrm{E}_{Q}\left[D_{0}\right]=100
$$

since $D_{0}$ is certain. It follows that

$$
\mathrm{E}_{Q}\left[\widetilde{D}_{1}\right]=120 \cdot 0.25+110 \cdot 0.75=112.5
$$

At time $t=2$ we get similarly

$$
\mathrm{E}_{Q}\left[\widetilde{D}_{2}\right]=150 \cdot 0.25^{2}+145 \cdot 2 \cdot 0.25 \cdot 0.75+100 \cdot 0.75^{2}=120
$$


Using both equations gives us

$$
\begin{aligned}
\widetilde{V}_{0}^{l}-\widetilde{V}_{0}^{u} & =\frac{0.1 \cdot 0.5 \cdot 100}{1+0.1}+\frac{0.1 \cdot 0.5 \cdot 112.5}{(1+0.1)^{2}}+\frac{0.1 \cdot 0.5 \cdot 120}{(1+0.1)^{3}} \\
& \approx 13.702 .
\end{aligned}
$$

3. Equation (3.11) implies

$$
\widetilde{V}_{t+1}^{l}=\widetilde{V}_{t+1}^{u}+\frac{\mathrm{E}_{Q}\left[\tau r_{f} \widetilde{D}_{t+1} \mid \mathcal{F}_{t+1}\right]}{1+r_{f}}+\ldots+\frac{\mathrm{E}_{Q}\left[\tau r_{f} \widetilde{D}_{T-1} \mid \mathcal{F}_{t+1}\right]}{\left(1+r_{f}\right)^{T-t-1}}
$$

Rule 4 gives

$$
\mathrm{E}_{Q}\left[\widetilde{V}_{t+1}^{l}-\widetilde{V}_{t+1}^{u} \mid \mathcal{F}_{t}\right]=\frac{\mathrm{E}_{Q}\left[\tau r_{f} \widetilde{D}_{t+1} \mid \mathcal{F}_{t}\right]}{1+r_{f}}+\ldots+\frac{\mathrm{E}_{Q}\left[\tau r_{f} \widetilde{D}_{T-1} \mid \mathcal{F}_{t}\right]}{\left(1+r_{f}\right)^{T-t-1}}
$$

or

$$
\frac{\mathrm{E}_{Q}\left[\widetilde{V}_{t+1}^{l}-\widetilde{V}_{t+1}^{u} \mid \mathcal{F}_{t}\right]}{1+r_{f}}=\frac{\mathrm{E}_{Q}\left[\tau r_{f} \widetilde{D}_{t+1} \mid \mathcal{F}_{t}\right]}{\left(1+r_{f}\right)^{2}}+\ldots+\frac{\mathrm{E}_{Q}\left[\tau r_{f} \widetilde{D}_{T-1} \mid \mathcal{F}_{t}\right]}{\left(1+r_{f}\right)^{T-t}}
$$

Adding $\frac{\tau r_{f} \widetilde{D}_{t}}{1+r_{f}}$ and taking into account that $\widetilde{D}_{t}$ is known at time $t$ (Rule 5) gives

$$
\begin{aligned}
\frac{\mathrm{E}_{Q}\left[\widetilde{V}_{t+1}^{l}-\widetilde{V}_{t+1}^{u} \mid \mathcal{F}_{t}\right]}{1+r_{f}}+\frac{\tau r_{f} \widetilde{D}_{t}}{1+r_{f}} & =\frac{\mathrm{E}_{Q}\left[\tau r_{f} \widetilde{D}_{t} \mid \mathcal{F}_{t}\right]}{1+r_{f}}+\ldots+\frac{\mathrm{E}_{Q}\left[\tau r_{f} \widetilde{D}_{T-1} \mid \mathcal{F}_{t}\right]}{\left(1+r_{f}\right)^{T-t}} \\
& =\widetilde{V}_{t}^{l}-\widetilde{V}_{t}^{u}
\end{aligned}
$$

and this is the claim.

We have shown that the value of the tax shield in $t+1$ and the value of the tax shield in $t$ are related by the above equation. Such a relation is sometimes called recursive. If $Q$ is unknown still an evaluation of the tax shield is possible if all $\widetilde{D}_{t}$ are known using this recursive equation.

4. From (3.11) we have

$$
\begin{aligned}
\widetilde{V}_{t}^{l} & =\widetilde{V}_{t}^{u}+\sum_{s=t}^{T-1} \frac{\tau r_{f} \mathrm{E}_{Q}\left[\widetilde{D}_{s} \mid \mathcal{F}_{t}\right]}{\left(1+r_{f}\right)^{s+1-t}} \\
& =\widetilde{V}_{t}^{u}+\sum_{s=t}^{T-1} \frac{\tau \mathrm{E}_{Q}\left[\widetilde{D}_{s} \mid \mathcal{F}_{t}\right]}{\left(1+r_{f}\right)^{s-t}}\left(1-\frac{1}{1+r_{f}}\right)
\end{aligned}
$$


and from this

$$
\begin{aligned}
\widetilde{V}_{t}^{l} & =\widetilde{V}_{t}^{u}+\sum_{s=t}^{T-1} \frac{\tau \mathrm{E}_{Q}\left[\widetilde{D}_{s} \mid \mathcal{F}_{t}\right]}{\left(1+r_{f}\right)^{s-t}}-\sum_{s=t}^{T-1} \frac{\tau \mathrm{E}_{Q}\left[\widetilde{D}_{s} \mid \mathcal{F}_{t}\right]}{\left(1+r_{f}\right)^{s+1-t}} \\
& =\widetilde{V}_{t}^{u}+\tau \widetilde{D}_{t}+\sum_{s=t+1}^{T-1} \frac{\tau \mathrm{E}_{Q}\left[\widetilde{D}_{s} \mid \mathcal{F}_{t}\right]}{\left(1+r_{f}\right)^{s-t}}-\sum_{s=t}^{T-1} \frac{\tau \mathrm{E}_{Q}\left[\widetilde{D}_{s} \mid \mathcal{F}_{t}\right]}{\left(1+r_{f}\right)^{s+1-t}} \\
& =\widetilde{V}_{t}^{u}+\tau \widetilde{D}_{t}+\sum_{s=t}^{T-1} \frac{\tau \mathrm{E}_{Q}\left[\widetilde{D}_{s+1} \mid \mathcal{F}_{t}\right]}{\left(1+r_{f}\right)^{s+1-t}}-\sum_{s=t}^{T-1} \frac{\tau \mathrm{E}_{Q}\left[\widetilde{D}_{s} \mid \mathcal{F}_{t}\right]}{\left(1+r_{f}\right)^{s+1-t}}
\end{aligned}
$$

which was to be shown (note that $\widetilde{D}_{T}=0$ ).

\subsubsection{Autonomous Financing}

1. We have

$$
\begin{aligned}
\mathrm{E}\left[\widetilde{F C F}_{t+1}^{l} \mid \mathcal{F}_{t}\right] & =\mathrm{E}\left[\widetilde{F C F}_{t+1}^{u}+\tau r_{f} \widetilde{D}_{t} \mid \mathcal{F}_{t}\right] \\
& =\mathrm{E}\left[\widetilde{F C F}_{t+1}^{u} \mid \mathcal{F}_{t}\right]+\tau r_{f} \widetilde{D}_{t} \\
& =\left(1+g_{t}\right){\widetilde{F C F_{t}}}^{u}+\tau r_{f} \widetilde{D}_{t} \\
& \neq\left(1+g_{t}\right) \underbrace{\left(\widetilde{F C F}_{t}^{u}+\tau r_{f} \widetilde{D}_{t-1}\right)}_{=\widetilde{F C F}_{t}^{l}} .
\end{aligned}
$$

For weak auto-regressive levered cash flows debt $\widetilde{D}_{t-1}$ must increase with the growth rate

$$
\widetilde{D}_{t}=\left(1+g_{t}\right) \widetilde{D}_{t-1}=\ldots=\left(1+g_{t}\right) \ldots\left(1+g_{1}\right) D_{0}
$$

and is therefore deterministic.

2. In the infinite example the value of the firm can be rearranged as follows:

$$
\begin{aligned}
\widetilde{V}_{t}^{l} & =\widetilde{V}_{t}^{u}+\sum_{s=t+1}^{\infty} \frac{\tau r_{f} D_{s}}{\left(1+r_{f}\right)^{s-t}} \\
& =\frac{\widetilde{F C F}_{t}^{u}}{k^{E, u}}+\tau r_{f} D_{t} \sum_{s=t+1}^{\infty} \frac{1}{\left(1+r_{f}\right)^{s-t}} \\
& =\frac{\widetilde{F C F}_{t}^{u}}{k^{E, u}}+\tau r_{f} D_{t} \frac{1}{r_{f}} \\
& =\frac{\widetilde{F C F}_{t}^{u}}{k^{E, u}}+\tau D_{t} .
\end{aligned}
$$


3. The dividend-price ratio of the levered firm is

$$
\begin{aligned}
d_{t}^{l} & =\frac{\widetilde{F C F}_{t}^{l}}{\widetilde{V}_{t}^{l}} \\
& =\frac{\widetilde{F C F}_{t}^{u}+\tau r_{f} D_{t-1}}{\widetilde{V}_{t}^{u}+\tau D_{t}} \\
& =k^{E, u} \frac{\widetilde{V}_{t}^{u}+\frac{r_{f}}{k^{E, u}} \tau D_{t-1}}{\widetilde{V}_{t}^{u}+\tau D_{t-1}} .
\end{aligned}
$$

To verify that this dividend ratio is stochastic we first realize that $\frac{r_{f}}{k^{E, u}} \neq 1$ since the cash flows are uncertain. Then, the function

$$
f(x):=\frac{x+\frac{r_{f}}{k^{E, u}} \tau D_{t-1}}{x+\tau D_{t-1}}
$$

is strictly monotone and hence $f\left(\widetilde{V}_{t}^{u}\right)$ will again be a random variable, i.e., if for two different states $\widetilde{V}_{t}^{u}(\omega)$ is different from $\widetilde{V}_{t}^{u}\left(\omega^{\prime}\right)$, it will be true that $d_{t}^{l}(\omega) \neq$ $d_{t}^{l}\left(\omega^{\prime}\right)$ and hence the dividend-price ratio is stochastic.

4. The conditional $Q$-probabilities are as in Fig. 3.1. From this, the unconditional probabilities can easily be evaluated: see Table 7.1 .

This yields the following $Q$-expected cash flows:

$$
\mathrm{E}_{Q}\left[\widetilde{F C F}_{1}^{l}\right]=96.6667, \quad \mathrm{E}_{Q}\left[\widetilde{F C F}_{2}^{l}\right]=97.4306, \quad \mathrm{E}_{Q}\left[\widetilde{F C F}_{3}^{l}\right]=95.7008
$$

From this, immediately

$$
V_{0}=\frac{\mathrm{E}_{Q}\left[\widetilde{F C F}_{1}^{l}\right]}{1+r_{f}}+\frac{\mathrm{E}_{Q}\left[\widetilde{F C F}_{2}^{l}\right]}{\left(1+r_{f}\right)^{2}}+\frac{\mathrm{E}_{Q}\left[\widetilde{F C F}_{3}\right]}{\left(1+r_{f}\right)^{3}} \approx 240.30
$$

as was to be shown.

Table 7.1 Unconditional $Q$-probabilities

\begin{tabular}{l|l}
\hline Time & $Q(\cdot)$ \\
\hline$t=1$ & $Q_{1}(u)=0.0833, Q_{1}(d)=0.9167$ \\
\hline$t=2$ & $Q_{2}(u u)=0.00347, Q_{2}(u d)=0.07986, Q_{2}(d u)=0.11458, Q_{2}(d d)=0.80208$ \\
\hline$t=3$ & $Q_{3}(u u u)=0.00130, Q_{3}(u u d)=0.00217, Q_{2}(u d u)=0.05657, Q_{2}(d u u)=0.08116$, \\
& $Q_{3}(u d d)=0.02329, Q_{3}(d u d)=0.08116, Q_{3}(d d u)=0.33420, Q_{3}(d d d)=0.46788$ \\
\hline
\end{tabular}


5. Due to (3.19),

$$
\begin{aligned}
\left(1+r_{f}\right) D_{2} & =\left(1+\widetilde{k}_{2}^{D, n o m}(d d)\right) D_{2}\left(1-Q_{3}(\mathrm{~d} \mid \mathrm{dd})\right)+\frac{\widetilde{F C F}_{3}^{u}(d d d)-\tau D_{2}}{1-\tau} Q_{3}(\mathrm{~d} \mid \mathrm{dd}) \\
1.1 \cdot D_{2} & \approx\left(1+\widetilde{k}_{2}^{D, n o m}(d d)\right) \cdot D_{2} \cdot(1-0.5833)+\frac{48.4-0.5 \cdot D_{2}}{1-0.5} 0.5833 \\
\widetilde{k}_{2}^{D, n o m}(d d) & \approx 3.04024-\frac{135.531}{D_{2}} .
\end{aligned}
$$

From this equation it is clear that there will be default if $D_{2} \geq 46.09$, otherwise $\widetilde{k}_{2}^{D, n o m}(d d)=10 \%$.

The cost of debt is given by its definition as an expected return under the subjective probability,

$$
\begin{aligned}
\widetilde{k}_{2}^{D}(d d) & =\frac{\left(1+\widetilde{k}_{2}^{D, n o m}\right) D_{2}\left(1-P_{3}(\mathrm{~d} \mid \mathrm{dd})\right)+\frac{1}{1-\tau}\left(\widetilde{F C F}_{3}^{u}(d d d)-\tau D_{2}\right) P_{3}(\mathrm{~d} \mid \mathrm{dd})}{D_{2}}-1 \\
\widetilde{k}_{2}^{D}(d d) & \approx \frac{\left(1+3.04024-\frac{135.531}{D_{2}}\right) \cdot D_{2} \cdot(1-0.5)+\frac{1}{1-0.5}\left(48.4-0.5 D_{2}\right) \cdot 0.5}{D_{2}}-1 \\
& \approx 0.52012-\frac{19.3654}{D_{2}}
\end{aligned}
$$

for $D_{2} \geq 46.09$, otherwise $\widetilde{k}_{2}^{D}(d d)=10 \%$.

Remark For very large $D_{2}$ there will be a default at state $\omega=d u u$ (and probably $u u d, u d u$ as well) which requires a more complicated evaluation that we will not present here.

\subsubsection{Financing Based on Market Values}

1. (a) We start with the value of the levered firm at $t=0$,

$$
\begin{aligned}
V_{0}^{l} & =\frac{\mathrm{E}\left[\widetilde{F C F}_{1}^{u}\right]}{1+W A C C}+\frac{\mathrm{E}\left[\widetilde{F C F}_{2}^{u}\right]}{(1+W A C C)^{2}}+\frac{\mathrm{E}\left[\widetilde{F C F}_{3}^{u}\right]}{(1+W A C C)^{3}} \\
& =\frac{100}{1+0.18}+\frac{110}{(1+0.18)^{2}}+\frac{121}{(1+0.18)^{3}} \approx 237.39 .
\end{aligned}
$$

The value at $t=1$ depends on the state of nature. We get

$$
\begin{aligned}
\widetilde{V}_{1}^{l}= & \frac{\mathrm{E}\left[\widetilde{F C F}_{2}^{u} \mid \mathcal{F}_{1}\right]}{1+W A C C}+\frac{\mathrm{E}\left[\widetilde{F C F}_{3}^{u} \mid \mathcal{F}_{1}\right]}{(1+W A C C)^{2}} \\
& = \begin{cases}\frac{121}{1+0.18}+\frac{133.1}{(1+0.18)^{2}} \approx 198.13 & \text { if up, } \\
\frac{99}{1+0.18}+\frac{108.9}{(1+0.18)^{2}} \approx 162.11 & \text { if down. }\end{cases}
\end{aligned}
$$


(b) This debt schedule implies the following leverage ratios:

$$
l_{0}=\frac{D_{0}}{V_{0}^{l}}=\frac{50}{237.39} \approx 21.06 \%
$$

and

$$
\widetilde{l}_{1}=\frac{\widetilde{D}_{1}}{\widetilde{V}_{1}^{l}}= \begin{cases}\frac{60}{198.13} \approx 30.28 \% & \text { if up } \\ \frac{40}{162.11} \approx 24.67 \% & \text { if down }\end{cases}
$$

The Miles-Ezzell formula must not be applied.

(c) The WACC textbook formula will give us the cost of equity of the levered firm since

$$
\begin{aligned}
\widetilde{W A C C}_{1}(u) & =\widetilde{k}_{1}^{E, l}(u)\left(1-\widetilde{l}_{1}(u)\right)+r_{f}(1-\tau) \widetilde{l}_{1}(u) \\
0.18 & \approx \widetilde{k}_{1}^{E, l}(u) \cdot(1-0.3028)+0.1 \cdot(1-0.5) \cdot 0.3028
\end{aligned}
$$

implying

$$
\widetilde{k}_{1}^{E, l}(u) \approx 23.65 \%
$$

Analogously one gets

$$
\widetilde{k}_{1}^{E, l}(d) \approx 22.26 \%
$$

The weighted average cost of capital type 1 can be evaluated using the TCF textbook formula

$$
\begin{aligned}
& \widetilde{k}_{1}^{\varnothing}(u)=\widetilde{k}_{1}^{E, l}(u)\left(1-\widetilde{l}_{1}(u)\right)+r_{f} \widetilde{l}_{1}(u) \\
& \widetilde{k}_{1}^{\varnothing}(u) \approx 0.2365 \cdot(1-0.3028)+0.1 \cdot 0.3028 \approx 19.52 \%
\end{aligned}
$$

and again

$$
\begin{aligned}
& \widetilde{k}_{1}^{\varnothing}(d)=\widetilde{k}_{1}^{E, l}(d)\left(1-\widetilde{l}_{1}(d)\right)+r_{f} \widetilde{l}_{1}(d) \\
& \widetilde{k}_{1}^{\varnothing}(d) \approx 0.2226 \cdot(1-0.2467)+0.1 \cdot 0.2467 \approx 19.24 \% .
\end{aligned}
$$

Note that we did all calculations using EXCEL and rounded the numbers only after all calculations were done. 
2. We have

$$
\begin{aligned}
\text { WACC }-k^{E, u}\left(1-\tau l_{0}\right) & =(1+\mathrm{WACC})-\left(1+k^{E, u}(1-\tau l)\right) \\
& =\left(1+k^{E, u}\right)\left(1-\frac{\tau r_{f}}{1+r_{f}} l\right)-\left(1+k^{E, u}(1-\tau l)\right) \\
& =\frac{\left(k^{E, u}-r_{f}\right) \tau l}{1+r_{f}}>0
\end{aligned}
$$

which was to be shown.

3. In this case we have

$$
\begin{aligned}
\widetilde{V}_{t}^{l} & =\sum_{s=t+1}^{\infty} \frac{\mathrm{E}\left[\widetilde{F C F}_{s}^{u} \mid \mathcal{F}_{t}\right]}{(1+W A C C)^{s-t}} \\
& =\sum_{s=t+1}^{\infty} \frac{\widetilde{F C F}_{t}^{u}}{(1+W A C C)^{s-t}} \\
& =\frac{\widetilde{F C F}_{t}^{u}}{W A C C} .
\end{aligned}
$$

Since

$$
\widetilde{V}_{t}^{u}=\frac{\widetilde{F C F}_{t}^{u}}{k^{E, u}}
$$

using the Miles-Ezzell formula gives

$$
\widetilde{V}_{t}^{l}=\frac{\widetilde{F C F}_{t}^{u}}{k^{E, u}} \frac{k^{E, u}}{\left(1+k^{E, u}\right)\left(1-\frac{\tau r_{f}}{1+r_{f}} l\right)-1}
$$

which gives the result after some rearranging.

4. We have

$$
1+W A C C_{t}=\left(1+k_{t}^{E, u}\right)\left(1-\frac{\tau r_{f}}{1+r_{f}} l_{t}\right)
$$

according to Miles-Ezzell. Now plug in

$$
1+r_{f}+\left(\mathrm{E}\left[\tilde{r}_{M}\right]-r_{f}\right) \beta_{t}^{W A C C}=\left(1+r_{f}+\left(\mathrm{E}\left[\tilde{r}_{M}\right]-r_{f}\right) \beta_{t}^{E, u}\right)\left(1-\frac{\tau r_{f}}{1+r_{f}} l_{t}\right)
$$


and this can be rearranged to

$$
\beta_{t}^{W A C C}=\beta_{t}^{E, u}\left(1-\frac{\tau r_{f}}{1+r_{f}} l_{t}\right)-\frac{\tau r_{f}}{\mathrm{E}\left[\tilde{r}_{M}\right]-r_{f}} l_{t} .
$$

\subsubsection{Financing Based on Book Values}

1. (a) First, since

$$
\widetilde{F C F}_{t+1}^{u}=F C F_{0}^{u}+\varepsilon_{1}+\ldots+\varepsilon_{t}
$$

and using the hint we know that the cash flows are normally distributed. They have expectation $F C F_{0}^{u}$ and variance $t$.

(b) This is easy, since

$$
\widetilde{V}_{t}^{u}=\frac{\widetilde{F C F}_{t}^{u}}{k^{E, u}}
$$

the value of the firm is normally distributed and has expectation $\frac{F C F_{0}^{u}}{k^{E, u}}$ and variance $\frac{t}{\left(k^{E, u}\right)^{2}}$.

(c) For the book value we have using (6.8) and the assumptions on the past of the firm

$$
\underline{V}_{t}^{l}=\underline{V}_{0}^{l}+\sum_{s=t-n+1}^{t} \frac{n-(t-s)}{n} \alpha \widetilde{F C F}_{s}^{u}
$$

Every summand $\frac{n-(t-s)}{n} \alpha \widetilde{F C F}_{s}^{u}$ is normally distributed with expectation $\frac{n-(t-s)}{n} \alpha F C F_{0}^{u}$ and variance $\left(\frac{n-(t-s)}{n} \alpha\right)^{2} t$. Hence, the whole sum is again normally distributed. It has expectation

$$
\underline{V}_{0}^{l}+\sum_{s=t-n+1}^{t} \frac{n-(t-s)}{n} \alpha F C F_{0}^{u}=\underline{V}_{0}^{l}+\frac{n+1}{2} \alpha F C F_{0}^{u}
$$

When evaluating the variance we have to take into account that the $\widetilde{F C F}_{s}^{u}$ are correlated. We assume $t>n$ for simplicity and get

$$
\begin{aligned}
& \operatorname{Var}\left[\sum_{s=t-n+1}^{t} \frac{n-(t-s)}{n} \alpha \widetilde{F C F}_{s}^{u}\right]=\alpha^{2} \operatorname{Var}\left[\sum_{s=t-n+1}^{t} \frac{n-(t-s)}{n} \sum_{r=1}^{s} \varepsilon_{r}\right] \\
& =\alpha^{2} \operatorname{Var}\left[\frac{1}{n} \sum_{s=1}^{t-n+1} \varepsilon_{s}+\frac{2}{n} \sum_{s=1}^{t-n+2} \varepsilon_{s}+\ldots+\frac{n}{n} \sum_{s=1}^{t} \varepsilon_{s}\right]
\end{aligned}
$$




$$
\begin{gathered}
=\alpha^{2} \operatorname{Var}\left[\sum_{s=1}^{t-n+1}\left\{\frac{1}{n}+\frac{2}{n}+\ldots+\frac{n}{n}\right\} \varepsilon_{s}+\left\{\frac{2}{n}+\ldots+\frac{n}{n}\right\} \varepsilon_{t-n+2}+\ldots+\left\{\frac{n}{n}\right\} \varepsilon_{t}\right] \\
=\alpha^{2} \operatorname{Var}\left[\sum_{s=1}^{t-n+1} \frac{n(n+1)}{2 n} \varepsilon_{s}+\frac{(n+2)(n+1-2)}{2 n} \varepsilon_{t-n+2}\right. \\
\left.+\frac{(n+3)(n+1-3)}{2 n} \varepsilon_{t-n+3}+\ldots+\frac{(n+n)(n+1-n)}{2 n} \varepsilon_{t}\right] .
\end{gathered}
$$

Since the $\varepsilon$ are pairwise independent it follows that the variance is equal to

$$
\begin{aligned}
\alpha^{2} & {\left[\sum_{s=1}^{t-n+1} \operatorname{Var}\left[\varepsilon_{s}\right] \frac{n^{2}(n+1)^{2}}{4 n^{2}}+\frac{(n+2)^{2}(n+1-2)^{2}}{4 n^{2}} \operatorname{Var}\left[\varepsilon_{t-n+2}\right]+\right.} \\
& \left.+\frac{(n+3)^{2}(n+1-3)^{2}}{4 n^{2}} \operatorname{Var}\left[\varepsilon_{t-n+3}\right]+\ldots+\frac{(n+n)^{2}(n+1-n)^{2}}{4 n^{2}} \operatorname{Var}\left[\varepsilon_{t}\right]\right] .
\end{aligned}
$$

Since the variance of all noise terms is 1 , this can be simplified to

$$
\begin{array}{r}
\alpha^{2}\left[\frac{n^{2}(n+1)^{2}(t-n)}{4 n^{2}}+\frac{(n+1)^{2}(n+1-1)^{2}}{4 n^{2}}+\frac{(n+2)^{2}(n+1-2)^{2}}{4 n^{2}}\right. \\
\left.+\frac{(n+3)^{2}(n+1-3)^{2}}{4 n^{2}}+\ldots+\frac{(n+n)^{2}(n+1-n)^{2}}{4 n^{2}}\right] .
\end{array}
$$

Although this is a complicated sum it can nevertheless be evaluated (for example, using Mathematica) and we get

$$
\alpha^{2}\left[\frac{n^{2}(n+1)^{2}(t-n)}{4 n^{2}}+\frac{(n+1)(1+2 n)\left(1+2 n+2 n^{2}\right)}{30 n}\right]
$$

and after simplification,

$$
\alpha^{2} \frac{(1+n)\left(2+15 \operatorname{tn}(n+1)+8 n-3 n^{2}-7 n^{3}\right)}{60 n} .
$$

This is the variance of the book value of the firm.

These evaluations show two interesting things:

- The expectation of the future book value does not depend on time. It stays constant at a level above the book value today.

- The variance does depend on time. With higher $t$ the variance increases, the increase is linear with slope $\alpha \frac{(n+1)^{2}}{4}$. 
2. The value of the firm that is financed by book value is given by

$$
\begin{aligned}
V_{0}^{l}(\text { finan. book value })= & V_{0}^{u}+\tau D_{0}+\frac{n r_{f}-1+\left(1+r_{f}\right)^{-n}}{n r_{f}} \tau \alpha \underline{l} V_{0}^{u} \\
= & \frac{100}{0.15}+0.34 \cdot 500 \\
& \quad+\frac{4 \cdot 0.05-1+(1+0.05)^{-4}}{4 \cdot 0.05} \cdot 0.34 \cdot 0.5 \cdot 0.7 \cdot \frac{100}{0.15}
\end{aligned}
$$

$\approx 845.672$.

The Miles-Ezzell formula for infinite lifetime (see problem 3 in Sect. 3.4.7) can be applied, but $l_{0}$ is not known (if $l_{0}$ were equal to $\underline{l}$, then book and market value would coincide). Hence,

$$
\begin{aligned}
& V_{0}^{l}(\text { finan. market value })=\frac{\mathrm{E}\left[\widetilde{F C F}_{t}^{u}\right]}{\left(1+k^{E, u}\right)\left(1-\frac{\tau r_{f}}{1+r_{f}} l_{0}\right)-1} \\
& =\frac{{\widetilde{F C F_{t}}}_{t}^{u}}{\left(1+k^{E, u}\right)\left(1-\frac{\tau r_{f}}{1+r_{f}} \frac{D_{0}}{V_{0}^{l}(\text { finan. market value })}\right)-1} \text {. }
\end{aligned}
$$

This can be rearranged to

$$
\begin{aligned}
V_{0}^{l}(\text { finan. market value }) & =\frac{\mathrm{E}\left[\widehat{F C F}_{t}^{u}\right]}{k^{E, u}}+\frac{\tau r_{f}}{1+r_{f}} \frac{1+k^{E, u}}{k^{E, u}} D_{0} \\
& =\frac{100}{0.15}+\frac{0.34 \cdot 0.05}{1+0.05} \frac{1+0.15}{0.15} 500 \\
& \approx 728.73 .
\end{aligned}
$$

Obviously, the difference is very big.

\subsubsection{Other Financing Policies}

1. First, if $n \rightarrow \infty$ and $T \rightarrow \infty$

$$
\lim _{n \rightarrow \infty} \delta^{n}=\lim _{T \rightarrow \infty} \delta^{T}=0
$$


since $\delta<1$. The same holds for $\gamma$ and $\frac{1}{1+r_{f}}$. Now Theorem 3.25 reduces to

$$
\begin{array}{r}
\lim _{T, n \rightarrow \infty} V_{0}^{l}=(1-0(1-\tau(1-0))) D_{0} \\
+(1-0(1-\tau(1-0))-\tau(1-0)) \frac{D i v}{r_{f}(1-\tau)} \\
+\left(0-0+\frac{0}{\frac{\gamma}{\delta}-1} \frac{k^{E, u}-g}{1+g}\right) \frac{V_{0}^{u}}{1-0}
\end{array}
$$

which was to be shown.

2. In a binomial model, the individual probabilities $Q$ can be determined (see Fig. 3.1).

(a) Using (3.11) this gives

$$
\begin{aligned}
V_{0}^{l} & =V_{0}^{u}+\left(\widetilde{D}_{1}(u) Q_{1}(u)+\widetilde{D}_{1}(d) Q_{1}(d)\right) \frac{\tau r_{f}}{\left(1+r_{f}\right)^{2}} \\
& =159.72+\left(\widetilde{D}_{1}(u) \cdot 0.083+\widetilde{D}_{1}(d) \cdot 0.917\right) \cdot 0.0281
\end{aligned}
$$

(b) Since

$$
100=\mathrm{E}\left[\widetilde{D}_{1}\right]=\frac{1}{2}\left(\widetilde{D}_{1}(d)+\widetilde{D}_{1}(u)\right)
$$

and since debt cannot be negative, the highest value of $V_{0}^{l}$ is achieved for

$$
\widetilde{D}_{1}(d)=200, \quad \widetilde{D}_{1}(u)=0 .
$$

This yields a firm value of 164.87 .

\subsection{Personal Income Tax}

\subsubsection{Unlevered and Levered Firms}

1. Due to Theorem 4.4, we get

$$
\begin{aligned}
\frac{\mathrm{E}_{Q}\left[\widetilde{F C F}_{1}^{u}\right]}{1+r_{f}(1-\tau)} & =\frac{\mathrm{E}\left[\widetilde{F C F}_{1}^{u}\right]}{1+k^{E, u}} \\
\frac{Q_{1}(u) \widetilde{F C F}_{1}^{u}(u)+Q_{1}(d) \widetilde{F C F}_{1}^{u}(d)}{1+r_{f}(1-\tau)} & =\frac{\mathrm{E}\left[\widetilde{F C F}_{1}^{u}\right]}{1+k^{E, u}} \\
\frac{Q_{1}(u) \cdot 110+Q_{1}(d) \cdot 90}{1.05} & =\frac{100}{1.2},
\end{aligned}
$$


and from this

$$
Q_{1}(u) \approx-0.125, \quad Q_{1}(d) \approx 1.125 .
$$

Any claim that pays one dollar after tax if $u p$ and nothing after tax if down must have a price of

$$
V_{0}^{u}=\frac{Q_{1}(u)}{1+r_{f}(1-\tau)}<0
$$

and this is an arbitrage opportunity. If $k^{E, u}=15 \%$ we get

$$
Q_{1}(u) \approx 0.0652, \quad Q_{1}(d) \approx 0.9348 .
$$

To evaluate $Q_{2}(d d), \ldots$ we concentrate on $t=1$. Analogously, we must have

$$
\frac{Q_{2}(u \mid u) \cdot 132+Q_{2}(d \mid u) \cdot 110}{1.05}=\frac{121}{1.15},
$$

which gives

$$
Q_{2}(u \mid u) \approx 0.0217, \quad Q_{2}(d \mid u) \approx 0.9783 .
$$

Now

$$
\begin{aligned}
& Q_{2}(u u)=Q_{1}(u) Q_{2}(u \mid u) \approx 0.0014 \\
& Q_{2}(u d)=Q_{1}(u) Q_{2}(u \mid d) \approx 0.0638
\end{aligned}
$$

and analogously

$$
Q_{2}(d u) \approx 0.1016, \quad Q_{2}(d d) \approx 0.8332 .
$$

2. Equation (4.4) implies

$$
\begin{array}{r}
\widetilde{V}_{t+1}^{l}=\widetilde{V}_{t+1}^{u}+\left(1-\tau^{D}\right) \widetilde{A}_{t+1}+\frac{\mathrm{E}_{Q}\left[\tau^{I} r_{f}\left(1-\tau^{D}\right) \widetilde{A}_{t+1} \mid \mathcal{F}_{t+1}\right]}{1+r_{f}\left(1-\tau^{I}\right)}+\ldots \\
+\frac{\mathrm{E}_{Q}\left[\tau^{I} r_{f}\left(1-\tau^{D}\right) \widetilde{A}_{T-1} \mid \mathcal{F}_{t+1}\right]}{\left(1+r_{f}\left(1-\tau^{I}\right)\right)^{T-t-1}} .
\end{array}
$$

Rule 4 gives

$$
\begin{gathered}
\mathrm{E}_{Q}\left[\widetilde{V}_{t+1}^{l}-\widetilde{V}_{t+1}^{u} \mid \mathcal{F}_{t}\right]=\mathrm{E}_{Q}\left[\left(1-\tau^{D}\right) \widetilde{A}_{t+1} \mid \mathcal{F}_{t}\right]+ \\
+\frac{\mathrm{E}_{Q}\left[\tau^{I} r_{f}\left(1-\tau^{D}\right) \widetilde{A}_{t+1} \mid \mathcal{F}_{t}\right]}{1+r_{f}\left(1-\tau^{I}\right)}+\ldots+\frac{\mathrm{E}_{Q}\left[\tau^{I} r_{f}\left(1-\tau^{D}\right) \widetilde{A}_{T-1} \mid \mathcal{F}_{t}\right]}{\left(1+r_{f}\left(1-\tau^{I}\right)\right)^{T-t-1}}
\end{gathered}
$$


or

$$
\begin{aligned}
\frac{\mathrm{E}_{Q}\left[\widetilde{V}_{t+1}^{l}-\widetilde{V}_{t+1}^{u}-\left(1-\tau^{D}\right) \widetilde{A}_{t+1} \mid \mathcal{F}_{t}\right]}{1+r_{f}\left(1-\tau^{I}\right)}= & \frac{\mathrm{E}_{Q}\left[\tau^{I} r_{f}\left(1-\tau^{D}\right) \widetilde{A}_{t+1} \mid \mathcal{F}_{t}\right]}{\left(1+r_{f}\left(1-\tau^{I}\right)\right)^{2}}+\ldots \\
& +\frac{\mathrm{E}_{Q}\left[\tau^{I} r_{f}\left(1-\tau^{D}\right) \widetilde{A}_{T-1} \mid \mathcal{F}_{t}\right]}{\left(1+r_{f}\left(1-\tau^{I}\right)\right)^{T-t}}
\end{aligned}
$$

Now adding $\frac{\mathrm{E}_{Q}\left[\tau^{I} r_{f}\left(1-\tau^{D}\right) \widetilde{A}_{t} \mid \mathcal{F}_{t}\right]}{1+r_{f}\left(1-\tau^{I}\right)}$ results in

$$
\begin{gathered}
\frac{\mathrm{E}_{Q}\left[\widetilde{V}_{t+1}^{l}-\widetilde{V}_{t+1}^{u} \mid \mathcal{F}_{t}\right]}{1+r_{f}\left(1-\tau^{I}\right)}+\frac{\mathrm{E}_{Q}\left[\tau^{I} r_{f}\left(1-\tau^{D}\right) \widetilde{A}_{t}-\left(1-\tau^{D}\right) \widetilde{A}_{t+1} \mid \mathcal{F}_{t}\right]}{1+r_{f}\left(1-\tau^{I}\right)}= \\
\frac{\mathrm{E}_{Q}\left[\tau^{I} r_{f}\left(1-\tau^{D}\right) \widetilde{A}_{t} \mid \mathcal{F}_{t}\right]}{1+r_{f}\left(1-\tau^{I}\right)}+\frac{\mathrm{E}_{Q}\left[\tau^{I} r_{f}\left(1-\tau^{D}\right) \widetilde{A}_{t+1} \mid \mathcal{F}_{t}\right]}{\left(1+r_{f}\left(1-\tau^{I}\right)\right)^{2}}+\ldots \\
+\frac{\mathrm{E}_{Q}\left[\tau^{I} r_{f}\left(1-\tau^{D}\right) \widetilde{A}_{T-1} \mid \mathcal{F}_{t}\right]}{\left(1+r_{f}\left(1-\tau^{I}\right)\right)^{T-t}}=\widetilde{V}_{t}^{l}-\widetilde{V}_{t}^{u}-\left(1-\tau^{D}\right) \widetilde{A}_{t} .
\end{gathered}
$$

Reshuffling gives

$$
\begin{aligned}
\widetilde{V}_{t}^{l}-\widetilde{V}_{t}^{u}= & \frac{\mathrm{E}_{Q}\left[\widetilde{V}_{t+1}^{l}-\widetilde{V}_{t+1}^{u} \mid \mathcal{F}_{t}\right]}{1+r_{f}\left(1-\tau^{I}\right)}+\frac{\left(1+r_{f}\right)\left(1-\tau^{D}\right)}{1+r_{f}\left(1-\tau^{I}\right)} \widetilde{A}_{t} \\
& -\frac{\mathrm{E}_{Q}\left[\left(1-\tau^{D}\right) \widetilde{A}_{t+1} \mid \mathcal{F}_{t}\right]}{1+r_{f}\left(1-\tau^{I}\right)} .
\end{aligned}
$$

3. We have

$$
\begin{aligned}
\widetilde{V}_{t}^{l} & =\widetilde{V}_{t}^{u}+\left(1-\tau^{D}\right) \widetilde{A}_{t}+\sum_{s=t}^{T} \frac{\tau^{I}\left(1-\tau^{D}\right) r_{f} \mathrm{E}_{Q}\left[\widetilde{A}_{s} \mid \mathcal{F}_{t}\right]}{\left(1+r_{f}\left(1-\tau^{I}\right)\right)^{s-t+1}} \\
& =\widetilde{V}_{t}^{u}+\left(1-\tau^{D}\right) \widetilde{A}_{t}+\frac{\tau^{I}\left(1-\tau^{D}\right) r_{f} \widetilde{A}_{t}}{1+r_{f}\left(1-\tau^{I}\right)}+\sum_{s=t+1}^{T} \frac{\tau^{I}\left(1-\tau^{D}\right) r_{f} \mathrm{E}_{Q}\left[\widetilde{A}_{s} \mid \mathcal{F}_{t}\right]}{\left(1+r_{f}\left(1-\tau^{I}\right)\right)^{s-t+1}} \\
& =\widetilde{V}_{t}^{u}+\frac{\left(1+r_{f}\right)\left(1-\tau^{D}\right)}{1+r_{f}\left(1-\tau^{I}\right)} \widetilde{A}_{t}+\sum_{s=t+1}^{T} \frac{\tau^{I}\left(1-\tau^{D}\right) r_{f} \mathrm{E}_{Q}\left[\widetilde{A}_{s} \mid \mathcal{F}_{t}\right]}{\left(1+r_{f}\left(1-\tau^{I}\right)\right)^{s-t+1}} \\
& =\widetilde{V}_{t}^{u}+\frac{\left(1+r_{f}\right)\left(1-\tau^{D}\right)}{1+r_{f}\left(1-\tau^{I}\right)} \widetilde{A}_{t}+\frac{\tau^{I}\left(1-\tau^{D}\right)}{1-\tau^{I}} \sum_{s=t+1}^{T} \frac{\mathrm{E}_{Q}\left[\widetilde{A}_{s} \mid \mathcal{F}_{t}\right]}{\left(1+r_{f}\left(1-\tau^{I}\right)\right)^{s-t}} \frac{\left(1-\tau^{I}\right) r_{f}}{1+r_{f}\left(1-\tau^{I}\right)}
\end{aligned}
$$




$$
\begin{aligned}
=\widetilde{V}_{t}^{u} & +\frac{\left(1+r_{f}\right)\left(1-\tau^{D}\right)}{1+r_{f}\left(1-\tau^{I}\right)} \widetilde{A}_{t}+\frac{\tau^{I}\left(1-\tau^{D}\right)}{1-\tau^{I}} \sum_{s=t+1}^{T} \frac{\mathrm{E}_{Q}\left[\widetilde{A}_{s} \mid \mathcal{F}_{t}\right]}{\left(1+r_{f}\left(1-\tau^{I}\right)\right)^{s-t}} \\
& +\left(1-\frac{1}{1+r_{f}\left(1-\tau^{I}\right)}\right) \\
=\widetilde{V}_{t}^{u} & +\frac{\left(1+r_{f}\right)\left(1-\tau^{D}\right)}{1+r_{f}\left(1-\tau^{I}\right)} \widetilde{A}_{t}+\frac{\tau^{I}\left(1-\tau^{D}\right)}{1-\tau^{I}} \sum_{s=t+1}^{T} \frac{\mathrm{E}_{Q}\left[\widetilde{A}_{s} \mid \mathcal{F}_{t}\right]}{\left(1+r_{f}\left(1-\tau^{I}\right)\right)^{s-t}} \\
& -\frac{\tau^{I}\left(1-\tau^{D}\right)}{1-\tau^{I}} \sum_{s=t+1}^{T} \frac{\mathrm{E}_{Q}\left[\widetilde{A}_{s} \mid \mathcal{F}_{t}\right]}{\left(1+r_{f}\left(1-\tau^{I}\right)\right)^{s-t+1}} \\
=\widetilde{V}_{t}^{u} & +\frac{\left(1+r_{f}\right)\left(1-\tau^{D}\right)}{1+r_{f}\left(1-\tau^{I}\right)} \widetilde{A}_{t}+\frac{\tau^{I}\left(1-\tau^{D}\right)}{1-\tau^{I}} \sum_{s=t+1}^{T} \frac{\mathrm{E}_{Q}\left[\widetilde{A}_{s} \mid \mathcal{F}_{t}\right]}{\left(1+r_{f}\left(1-\tau^{I}\right)\right)^{s-t}} \\
& -\frac{\tau^{I}\left(1-\tau^{D}\right)}{1-\tau^{I}} \sum_{s=t+2}^{T} \frac{\mathrm{E}_{Q}\left[\widetilde{A}_{s-1} \mid \mathcal{F}_{t}\right]}{\left(1+r_{f}\left(1-\tau^{I}\right)\right)^{s-t}}
\end{aligned}
$$

using $\widetilde{A}_{T}=0$. Rearranging gives

$$
\begin{aligned}
\widetilde{V}_{t}^{l}=\widetilde{V}_{t}^{u} & +\frac{\left(1+r_{f}\right)\left(1-\tau^{D}\right)}{1+r_{f}\left(1-\tau^{I}\right)} \widetilde{A}_{t}+\frac{\tau^{I}\left(1-\tau^{D}\right)}{1-\tau^{I}} \sum_{s=t+1}^{T} \frac{\mathrm{E}_{Q}\left[\widetilde{A}_{s} \mid \mathcal{F}_{t}\right]}{\left(1+r_{f}\left(1-\tau^{I}\right)\right)^{s-t}} \\
& -\frac{\tau^{I}\left(1-\tau^{D}\right)}{1-\tau^{I}} \sum_{s=t+1}^{T} \frac{\mathrm{E}_{Q}\left[\widetilde{A}_{s-1} \mid \mathcal{F}_{t}\right]}{\left(1+r_{f}\left(1-\tau^{I}\right)\right)^{s-t}}+\frac{\tau^{I}\left(1-\tau^{D}\right)}{1-\tau^{I}} \frac{\widetilde{A}_{t}}{1+r_{f}\left(1-\tau^{I}\right)}
\end{aligned}
$$

and this is equivalent to

$$
\widetilde{V}_{t}^{l}=\widetilde{V}_{t}^{u}+\frac{1-\tau^{D}}{1-\tau^{I}} \widetilde{A}_{t}+\frac{\tau^{I}\left(1-\tau^{D}\right)}{1-\tau^{I}} \sum_{s=t+1}^{T} \frac{\mathrm{E}_{Q}\left[\widetilde{A}_{s}-\widetilde{A}_{s-1} \mid \mathcal{F}_{t}\right]}{\left(1+r_{f}\left(1-\tau^{I}\right)\right)^{s-t}} .
$$

\subsubsection{Excursus: Cost of Equity and Tax Rate}

1. This follows from

$$
\begin{aligned}
k^{\text {post-tax }} & =\frac{\mathrm{E}\left[\widetilde{G C F}_{t+1}+\widetilde{V}_{t+1}^{u}-\tau\left(\widetilde{G C F}_{t+1}+\widetilde{V}_{t+1}^{u}-\widetilde{V}_{t}^{u}\right) \mid \mathcal{F}_{t}\right]}{\widetilde{V}_{t}^{u}} \\
& =(1-\tau) \frac{\mathrm{E}\left[\widetilde{G C F}_{t+1}+\widetilde{V}_{t+1}^{u} \mid \mathcal{F}_{t}\right]}{\widetilde{V}_{t}^{u}} \\
& =(1-\tau) k^{\text {pre-tax }} .
\end{aligned}
$$


2. We have

$$
\begin{aligned}
\widetilde{V}_{t}^{u} & =\sum_{s=t+1}^{\infty} \frac{\mathrm{E}_{Q}\left[\widetilde{G C F}_{s}(1-\tau) \mid \mathcal{F}_{t}\right]}{\left(1+r_{f}(1-\tau)\right)^{s-t}} \\
& =\sum_{s=t+1}^{\infty} \frac{\mathrm{E}_{Q}\left[\widetilde{G C F_{s}} \mid \mathcal{F}_{t}\right]}{\left(1+r_{f}\right)^{s-t}} \frac{(1-\tau)\left(1+r_{f}\right)^{s-t}}{\left(1+r_{f}(1-\tau)\right)^{s-t}} \\
& =\sum_{s=t+1}^{\infty} \frac{\mathrm{E}\left[\widetilde{G C F_{s}} \mid \mathcal{F}_{t}\right]}{\left(1+k^{\text {pre-tax }^{s-t}}\right.} \frac{(1-\tau)\left(1+r_{f}\right)^{s-t}}{\left(1+r_{f}(1-\tau)\right)^{s-t}} \\
& =\sum_{s=t+1}^{\infty} \frac{\widetilde{G C F}_{t}}{\left(1+k^{\text {pre-tax }^{s-t}}\right.} \frac{(1-\tau)\left(1+r_{f}\right)^{s-t}}{\left(1+r_{f}(1-\tau)\right)^{s-t}} \\
& =\frac{(1-\tau) \widetilde{G C F}_{t}}{\left(1+k^{\left.\mathrm{pre}^{\mathrm{tax}}\right)\left(1+r_{f}(1-\tau)\right)}-1\right.}
\end{aligned}
$$

and this is obviously different from

$$
\frac{(1-\tau) \widetilde{G C F}_{t}}{k^{\operatorname{pre}-\operatorname{tax}}(1-\tau)} .
$$

3. (a) As in Sect. 3.6.1 we get

$$
\begin{aligned}
\frac{\mathrm{E}_{Q}\left[\widetilde{G C F}_{1}\right]}{1+r_{f}} & =\frac{\mathrm{E}\left[\widetilde{G C F}_{1}\right]}{1+k} \\
\frac{Q_{1}(u) \widetilde{G C F}_{1}(u)+Q_{1}(d) \widetilde{G C F}_{1}(d)}{1+r_{f}} & =\frac{\mathrm{E}\left[\widetilde{G C F}_{1}\right]}{1+k} \\
\frac{Q_{1}(u) \cdot 110+Q_{1}(d) \cdot 90}{1.05} & =\frac{100}{1.15},
\end{aligned}
$$

and hence

$$
Q_{1}(u) \approx 0.0652, \quad Q_{1}(d) \approx 0.93478
$$


(b) If we look at the second company, the gross cash flows are different and we get

$$
\begin{aligned}
\frac{\mathrm{E}_{Q}\left[\overline{G C F}_{1}^{\prime}\right]}{1+r_{f}} & =\frac{\mathrm{E}\left[\overline{G C F}_{1}^{\prime}\right]}{1+k^{\prime}} \\
\frac{Q_{1}(u) \widetilde{G C F}_{1}^{\prime}(u)+Q_{1}(d){\widetilde{G C F_{1}}}_{1}^{\prime}(d)}{1+r_{f}} & =\frac{\mathrm{E}\left[{\widetilde{G C F_{1}^{\prime}}}_{1}^{\prime}\right.}{1+k^{\prime}} \\
\frac{0.0652 \cdot 120+0.93478 \cdot 80}{1.05} & \approx \frac{100}{1+k^{\prime}},
\end{aligned}
$$

and hence

$$
k^{\prime} \approx 27.105 \%
$$

(c) This is the same calculation except that we have to add taxes:

$$
\begin{aligned}
\frac{\mathrm{E}_{Q}\left[(1-\tau) \overline{G C F}_{1}\right]}{1+r_{f}(1-\tau)} & =\frac{\mathrm{E}\left[(1-\tau) \widetilde{G C F}_{1}\right]}{1+k(1-\tau)} \\
\frac{Q_{1}(u)(1-\tau) \widetilde{G C F}_{1}(u)+Q_{1}(d)(1-\tau) \widetilde{G C F}_{1}(d)}{1+r_{f}(1-\tau)} & =\frac{\mathrm{E}\left[(1-\tau) \widetilde{G C F}_{1}\right]}{1+k(1-\tau)} \\
\frac{Q_{1}(u)(1-\tau) 110+Q_{1}(d)(1-\tau) 90}{1+0.05(1-\tau)} & =\frac{100(1-\tau)}{1+0.15(1-\tau)},
\end{aligned}
$$

and hence

$$
Q_{1}(u) \approx 2.8333 \frac{(0.176471+\tau)}{7.6667-\tau}, \quad Q_{1}(d)=1-Q_{1}(u) .
$$

(d) The calculation is as above,

$$
\begin{aligned}
\frac{\mathrm{E}_{Q}\left[(1-\tau) \widetilde{G C F}_{1}\right]}{1+r_{f}(1-\tau)} & =\frac{\mathrm{E}\left[(1-\tau) \widetilde{G C F}_{1}\right]}{1+k^{\prime}(1-\tau)} \\
\frac{Q_{1}(u)(1-\tau) \widetilde{G C F}_{1}(u)+Q_{1}(d)(1-\tau) \widetilde{G C F}_{1}(d)}{1+r_{f}(1-\tau)} & =\frac{\mathrm{E}\left[(1-\tau) \widetilde{G C F}_{1}\right]}{1+k^{\prime}(1-\tau)} \\
\frac{Q_{1}(u)(1-\tau) 120+Q_{1}(d)(1-\tau) 80}{1+0.05(1-\tau)} & =\frac{100(1-\tau)}{1+15.5 \%(1-\tau)},
\end{aligned}
$$


and hence

$$
Q_{1}(u) \approx 1.1667 \frac{(1.85714+\tau)}{7.6667-\tau}, \quad Q_{1}(d)=1-Q_{1}(u)
$$

This is different from the result in $3 \mathrm{c}$.

\subsubsection{Retention Policies}

1. From (4.4) it follows that the market value is maximized if the retentions are as large as possible. Since necessarily

$$
\widetilde{A_{3}}=0
$$

we only look at $\widetilde{A_{1}}$ and $\widetilde{A_{2}}$. We will determine the highest possible retention and evaluate the corresponding market value of the firm.

If the company maintains its highest possible retention, the cash flows to the shareholders are zero. From this

$$
\widetilde{A}_{1}:=\frac{1}{1-\tau^{D}} \widetilde{F C F}_{1}^{u}, \quad \widetilde{A}_{2}:=\left(1+r_{f}\right){\widetilde{A_{1}}}+\frac{1}{1-\tau^{D}} \widetilde{F C F}_{2}^{u} .
$$

We put this into (4.4) and get, using Theorem 4.4,

$$
\begin{aligned}
& V_{0}^{l}=V_{0}^{u}+\frac{\tau^{I} r_{f}\left(1-\tau^{D}\right) \mathrm{E}_{Q}\left[\widetilde{A}_{1}\right]}{\left(1+r_{f}\left(1-\tau^{I}\right)\right)^{2}}+\frac{\tau^{I} r_{f}\left(1-\tau^{D}\right) \mathrm{E}_{Q}\left[\widetilde{A}_{2}\right]}{\left(1+r_{f}\left(1-\tau^{I}\right)\right)^{3}} \\
& =V_{0}^{u}+\frac{\tau^{I} r_{f}\left(1-\tau^{D}\right) \mathrm{E}_{Q}\left[\frac{\widetilde{F C F}_{1}^{u}}{1-\tau^{D}}\right]}{\left(1+r_{f}\left(1-\tau^{I}\right)\right)^{2}}+\frac{\tau^{I} r_{f}\left(1-\tau^{D}\right) \mathrm{E}_{Q}\left[\frac{\left(1+r_{f}\right) \widetilde{F C F}_{1}^{u}+\widetilde{F C F}_{2}^{u}}{1-\tau^{D}}\right]}{\left(1+r_{f}\left(1-\tau^{I}\right)\right)^{3}} \\
& =V_{0}^{u}+\frac{\tau^{I} r_{f} \mathrm{E}_{Q}\left[\widetilde{F C F}_{1}^{u}\right]}{\left(1+r_{f}\left(1-\tau^{I}\right)\right)^{2}}+\frac{\tau^{I} r_{f}\left(1+r_{f}\right) \mathrm{E}_{Q}\left[\widetilde{F C F}_{1}^{u}\right]}{\left(1+r_{f}\left(1-\tau^{I}\right)\right)^{3}}+\frac{\tau^{I} r_{f} \mathrm{E}_{Q}\left[\widetilde{F C F}_{2}^{u}\right]}{\left(1+r_{f}\left(1-\tau^{I}\right)\right)^{3}} \\
& =V_{0}^{u}+\frac{\tau^{I} r_{f} \mathrm{E}\left[\widetilde{F C F}_{1}^{u}\right]}{\left(1+k^{E, u}\right)\left(1+r_{f}\left(1-\tau^{I}\right)\right)}+\frac{\tau^{I} r_{f}\left(1+r_{f}\right) \mathrm{E}\left[\widetilde{F C F}_{1}^{u}\right]}{\left(1+k^{E, u}\right)\left(1+r_{f}\left(1-\tau^{I}\right)\right)^{2}} \\
& +\frac{\tau^{I} r_{f} \mathrm{E}\left[\widetilde{F C F}_{2}^{u}\right]}{\left(1+k^{E, u}\right)^{2}\left(1+r_{f}\left(1-\tau^{I}\right)\right)}
\end{aligned}
$$




$$
\begin{aligned}
\approx 249.692+ & \frac{0.5 \cdot 0.1 \cdot 100}{(1+0.15) \cdot(1+0.1 \cdot(1-0.5))} \\
& +\frac{0.5 \cdot 0.1 \cdot(1+0.1) \cdot 100}{(1+0.15) \cdot(1+0.1 \cdot(1-0.5))^{2}} \\
& \quad+\frac{0.5 \cdot 0.1 \cdot 110}{(1+0.15)^{2} \cdot(1+0.1 \cdot(1-0.5))}
\end{aligned}
$$

$\approx 260.061$.

2. With retention in riskless assets (4.3) reads

$$
\mathrm{E}_{Q}\left[\widetilde{F C F}_{t}^{l}-\widetilde{F C F}_{t}^{u} \mid \mathcal{F}_{t-1}\right]=\left(1-\tau^{I}\right)\left(1+r_{f}\right) \widetilde{A}_{t-1}-\left(1-\tau^{I}\right) \mathrm{E}_{Q}\left[\widetilde{A}_{t} \mid \mathcal{F}_{t-1}\right] .
$$

Following Eq. (4.4) we get

$$
\begin{gathered}
\widetilde{V}_{t}^{l}=\widetilde{V}_{t}^{u}+\frac{\left(1-\tau^{I}\right) \mathrm{E}_{Q}\left[\left(1+r_{f}\right) \widetilde{A}_{t}-\widetilde{A}_{t+1} \mid \mathcal{F}_{t}\right]}{1+r_{f}\left(1-\tau^{I}\right)}+\ldots \\
+\frac{\left(1-\tau^{I}\right) \mathrm{E}_{Q}\left[\left(1+r_{f}\right) \widetilde{A}_{T-2}-\widetilde{A}_{T-1} \mid \mathcal{F}_{t}\right]}{1+r_{f}\left(1-\tau^{I}\right)^{T-t-1}} \\
+\frac{\left(1-\tau^{I}\right) \mathrm{E}_{Q}\left[\left(1+r_{f}\right) \widetilde{A}_{T-1} \mid \mathcal{F}_{t}\right]}{\left(1+r_{f}\left(1-\tau^{I}\right)\right)^{T-t}} .
\end{gathered}
$$

After some minimal reshuffling the following results:

$$
\begin{aligned}
& \widetilde{V}_{t}^{l}=\widetilde{V}_{t}^{u}+ \frac{\left(1-\tau^{I}\right)\left(1+r_{f}\right) \widetilde{A}_{t}}{1+r_{f}\left(1-\tau^{I}\right)} \\
&+ \frac{\mathrm{E}_{Q}\left[\frac{\left(1+r_{f}\right)\left(1-\tau^{I}\right)}{1+r_{f}\left(1-\tau^{I}\right)} \widetilde{A}_{t+1}-\left(1-\tau^{I}\right) \widetilde{A}_{t+1} \mid \mathcal{F}_{t}\right]}{1+r_{f}\left(1-\tau^{I}\right)}+\ldots \\
&+\frac{\mathrm{E}_{Q}\left[\frac{\left(1+r_{f}\right)\left(1-\tau^{I}\right)}{1+r_{f}\left(1-\tau^{I}\right)} \widetilde{A}_{T-1}-\left(1-\tau^{I}\right) \widetilde{A}_{T-1} \mid \mathcal{F}_{t}\right]}{\left(1+r_{f}\left(1-\tau^{I}\right)\right)^{T-t}}
\end{aligned}
$$


This brings us to the conclusion,

$$
\begin{aligned}
\widetilde{V}_{t}^{l}=\widetilde{V}_{t}^{u}+\left(1-\tau^{I}\right) \widetilde{A}_{t}+\frac{\tau^{I}\left(1-\tau^{I}\right) r_{f} \mathrm{E}_{Q}\left[\widetilde{A}_{t} \mid \mathcal{F}_{t}\right]}{1+r_{f}\left(1-\tau^{I}\right)}+\ldots \\
+\frac{\tau^{I}\left(1-\tau^{I}\right) r_{f} \mathrm{E}_{Q}\left[\widetilde{A}_{T-1} \mid \mathcal{F}_{t}\right]}{\left(1+r_{f}\left(1-\tau^{I}\right)\right)^{T-t}} .
\end{aligned}
$$

In case of autonomous retention, with an eternally living firm this simply yields

$$
\widetilde{V}_{t}^{l}=\widetilde{V}_{t}^{u}+A
$$

\subsection{Corporate and Personal Income Tax}

1. We get

$$
\begin{aligned}
\widetilde{V}_{t}^{l} & =\widetilde{V}_{t}^{u}+\frac{\left(1-\tau^{D}\right)\left(1-\tau^{C}\right)}{1-\tau^{I}} A+\tau^{C} D \\
& =500+\frac{(1-0.5) \cdot(1-0.5)}{1-0.5} 10+0.5 \cdot 100 \\
& =555 .
\end{aligned}
$$

Open Access This chapter is licensed under the terms of the Creative Commons Attribution 4.0 International License (http://creativecommons.org/licenses/by/4.0/), which permits use, sharing, adaptation, distribution and reproduction in any medium or format, as long as you give appropriate credit to the original author(s) and the source, provide a link to the Creative Commons licence and indicate if changes were made.

The images or other third party material in this chapter are included in the chapter's Creative Commons licence, unless indicated otherwise in a credit line to the material. If material is not included in the chapter's Creative Commons licence and your intended use is not permitted by statutory regulation or exceeds the permitted use, you will need to obtain permission directly from the copyright holder.

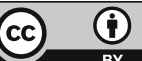

